\title{
Perceptual prioritisation of self-associated voices
}

Bryony Payne ${ }^{1}$, Nadine Lavan ${ }^{1}$ Sarah Knight ${ }^{2}$ \& Carolyn McGettigan ${ }^{1}$

${ }^{1}$ Department of Speech, Hearing and Phonetic Sciences, University College London, United Kingdom

${ }^{2}$ Department of Psychology, University of York, United Kingdom

\author{
Author Note \\ Correspondence concerning this article should be addressed to Bryony \\ Payne, Department of Speech, Hearing and Phonetic Sciences, University College \\ London, London, WC1N 1PF. \\ E-mail: bryony.payne.16@ucl.ac.uk
}

This work was supported by a Research Leadership Award from the Leverhulme Trust (RL-2016-013) awarded to Prof C McGettigan.

${ }^{* *}$ This is a pre-print of a manuscript that has been submitted for peer review**

Data and materials for this study can be accessed via the web links below:

Experiment 1: https://osf.io/hg3w6/

Experiment 2: https://osf.io/r6g2m/

Experiment 3: https://osf.io/wz2am/ 


\begin{abstract}
Information associated with the self is prioritised relative to information associated with others and is therefore processed more quickly and accurately. Across three experiments, we examined whether a new externally-generated voice could become associated with the self and thus be prioritised in perception.

In the first experiment, participants learned associations between three unfamiliar voices and three identities (self, friend, other). Participants then made speeded judgements of whether voice-identity pairs were correctly matched, or not. A clear selfprioritisation effect was found, with participants showing quicker and more accurate responses to the self-associated voice relative to either the friend- or other-associated voice.
\end{abstract}

In two further experiments, we tested whether this prioritisation effect increased if the self-associated voice was gender-matched to the identity of the participant (Experiment 2) or if the self-voice was chosen by the participant (Experiment 3). Gender-matching did not significantly influence prioritisation; the self-voice was similarly prioritised when it matched the gender identity of the listener as when it did not. However, we observed that choosing the self-voice did interact with prioritisation (Experiment 3); the self-voice became more prominent, via lesser prioritisation of the other identities, when the self-voice was chosen relative to when it was not.

Our findings have implications for the design and selection of individuated synthetic voices used for assistive communication devices, suggesting that agency in choosing a new vocal identity may modulate the distinctiveness of that voice relative to others.

Keywords: self prioritisation effect; self-bias; self-voice; vocal identity; voice synthesis. 


\section{Perceptual prioritisation of self-associated voices}

\section{Introduction}

Voice is central to the dynamic construction of the self and is therefore of great personal and social importance. A speaker's unique voice pattern conveys a wealth of information about their physical self to a listener, including their age, gender, health, and affective state (Kreiman \& Sidtis, 2011). A speaker also has extensive control over the dynamic use of their vocal apparatus, and is able to flexibly and deliberately modulate the acoustics of their voice (Hughes, Mogilski, \& Harrison, 2014; McGettigan et al., 2013; McGettigan \& Scott, 2014) to fulfil diverse communicative goals according to changes in social demands and the acoustic environment.

There is evidence that speakers are implicitly biased towards their own voice, such that they evaluate it as more attractive than the voices of others (Hughes \& Harrison, 2013). This bias appears in spite of the typically negative reaction that occurs when a speaker is aware of hearing their voice on a recording (Holzman \& Rousey, 1966), which suggests that the bias arises from an implicit egotism and, by extension, a preference for their voice as a self-associated and self-generated signal. It remains unclear, however, whether vocal stimuli need to be self-generated in order to elicit a self-bias. This study therefore asks whether an externally-generated vocal stimulus, such as another's voice, can become associated with the self and, in turn, elicit a processing bias.

This question is of direct relevance to patients with vocal impairments who cannot use their own vocal apparatus to produce sound but instead come to rely on a non-selfgenerated voice as their primary means of communication. Synthesized voices provided by augmentative and alternative communication (AAC) devices are often unnatural-sounding, lacking in emotional prosody and slower than self-produced speech. AAC users report feeling like they present a distorted representation of themselves, which can decrease the desire to engage in social interaction and contribute to the loss of a social identity (Nathanson, 2017). Only recently has the moral case for more personalised voices been considered, with companies such as VocaliD (www.vocalid.ai) now providing a selection of voices from which AAC users might choose a voice that better conveys their self-identity. 
It is important therefore to ask whether a new externally-generated voice can become associated with the self and elicit a self-bias. However, measuring the boundaries of the self has previously relied either on wholly subjective self-reports whereby participants explicitly evaluate themselves against others (see Symons \& Johnston, 1997) or on comparisons of processing biases between overly familiar self-associated stimuli, such as the true self-voice, and unfamiliar, non-self voices. Inherently, these approaches cannot address the problem of whether a new, unfamiliar self-voice can become self-associated and elicit a bias. Further, these approaches face a problematic confound of stimulus familiarity that makes it difficult to disentangle the potential bias for self-associated information from the familiarity of that information. More recent methodologies have therefore focused on creating paradigms that are both independent of stimulus familiarity and provide a more robust measure of selfbias (Cunningham, Turk, Macdonald, \& Macrae, 2008; Sui, He, \& Humphreys, 2012).

Sui et al. (2012) introduced a socio-associative perceptual matching task which required participants to form rapid new associations between themselves and arbitrary objects. Participants were asked to form associations between three geometric shapes (a circle, a triangle, and a square) and three identity labels (you, friend, stranger). For example, the participants were told: 'the circle represents you, the square a friend, and the triangle, a stranger'. These three shape-identity associations were thus established as 'matched' pairs. In the main task, participants were presented with pairings, each comprising one of the shapes and one of the identity labels, and asked to judge whether or not the two stimuli were correctly matched. The results consistently showed a clear perceptual bias towards the shape associated with the self, eliciting faster and more accurate responses compared to the shapes associated with either the friend or stranger (Sui et al., 2012).

Sui et al. have thus provided key insights into the nature of self-bias. Specifically, that the process of referring a stimulus to the self serves to form a rapid new selfassociation. Further, in becoming self-associated, the stimulus is assigned increased personal significance and becomes perceptually more salient. Neurally, this new selfassociation activates increased functional connectivity between the ventral medial prefrontal cortex, an area linked to self-representation, and the left posterior superior 
temporal sulcus, an area associated with social attention (Sui, Rotshtein and Humphreys, 2013). Thus, the social salience for the self-associated stimulus is moderated and it becomes perceptually prioritised. This processing bias is termed the self-prioritisation effect (Sui et al., 2012).

The ability to link new external stimuli to the self has also been explored in relation to memory. Cunningham et al. (2008; also, Cunningham, Brady-Van den Bos \& Turk, 2011) demonstrate that a stimulus can be encompassed within the self by taking ownership of it. Specifically, a collection of food items, represented pictorially, was divided into either the participant's shopping basket or another's basket. Each participant was told that they would own whichever food items were in their basket at the end. In a surprise test of memory recall, participants were more likely to remember the items they 'owned' than the items that had been placed in another's basket, despite the items being similar and the ownership being imaginary. This suggests that once an item or stimulus is associated with the self via ownership, encoding of that item is enhanced. This process may operate similarly to the self-prioritisation effect demonstrated by Sui et al. (2012).

Items that we own are also perceived as having more positive traits than similar items belonging to another. Gregg, Mahadevan, and Sedikides (2017) gave participants arbitrary ownership of an abstract concept by telling them 'you have a theory about...' while they gave other participants an abstract concept that was 'owned' by someone else, e.g. 'Alex has a theory about...'. When assessed, the participants who 'owned' the theory were more biased towards it; they were more likely to believe their theory was true, and also endorsed it more strongly than the participants given Alex's theory. This demonstrates that self-bias from affective-ownership can extend beyond concrete visual stimuli to abstract ideas. Although this positivity bias may reflect the consequence of encoding a stimulus to the self, rather than the mechanism, it demonstrates that the self can be extended to multiple different types of information at different levels of processing, all of which accrue a processing advantage.

Furthermore, Schäfer, Wesslein, Spence, Wentura and Frings (2016), have demonstrated that the bias for self-associated stimuli also holds for other sensory modalities, including touch and audition. Schäfer et al. (2016) replicated the perceptual 
matching paradigm using an adapted version with auditory or tactile stimulus cues. In the auditory associative task participants were asked to associate three neutrallyvalenced pure tones with the three identities of self, friend, and other. At test, results showed a comparable self-prioritisation effect for the self-associated auditory stimuli. This study thus provides the first evidence that self-prioritisation processes operate across the senses, and that we are able to incorporate a diverse range of sensory stimuli into the self.

Finally, the flexibility with which we can extend the self is further exemplified by Payne, Tsakiris, and Maister (2017), who asked participants to form new self-associations to three unfamiliar faces using the perceptual matching task. Payne et al. (2017) demonstrated that participants could successfully form an association to a new selfface, with results again showing prioritisation of faces arbitrarily tagged as 'self', relative to faces tagged as belonging to friends or strangers. This result is surprising given that faces are not neutral stimuli akin to arbitrary geometric shapes. Rather, our own face is one of the most salient pieces of self-associated information we have and, importantly, unfamiliar faces should typically be processed as other-associated purely by virtue of not being our own. If multifarious pieces of self-associated information together give rise to our self-identity - an identity that is recognisably distinct from another's identity - the incorporation of a new self-face into the representation of self seems to blur the boundary of self and other.

In demonstrating that self-bias can be attributed to an other's face, Payne et al.'s study provides the closest test case for which complex, other-related, signals can be accepted as part of the self. Voices are, like faces, highly social and identifying signals, yet they differ in that they require self-generated actions in order to exist; it is only through the varied, idiosyncratic, and deliberate use of our voice that we can share it with others to express our self-identity. Thus, to demonstrate that an unfamiliar voice generated by another person can become associated with our self and be perceptually prioritised would be a significant addition to our understanding of the self and the flexibility of our self-representation.

Here, we present three experiments using the paradigm introduced by Sui et al. (2012) to test whether the self-prioritisation effect is found for unfamiliar voice identities. In 
Experiment 1, we establish that the effect can indeed be observed for voices as has been previously reported for other stimulus categories. In Experiments 2 and 3, we explore whether properties of the voice stimuli (i.e. matching the participant's gender identity vs not) and the method of assignment to the self (i.e. choosing the self-voice vs having no choice) would modulate the effect. These questions relating to how a voice sounds, and how it is chosen, are directly pertinent to the applied context of AAC devices; however, these are also novel questions that allow us to expand upon the existing knowledge of the self-prioritisation effect for non-self-generated, social stimuli. All three experiments, including the planned analyses and predictions for each, were pre-registered via the Open Science Framework (see links below).

\section{Experiment 1}

In the first study, we asked whether a new, externally-generated voice could become associated with the self and prioritised in perception relative to voices associated with either a friend or a stranger. This experiment was preregistered via the Open Science Framework (https://osf.io/hg3w6/).

\subsection{Hypothesis}

Within the perceptual matching paradigm, listeners will have quicker reaction times and greater recognition accuracy for the new self-voice compared to either the friendvoice or stranger-voice, revealing a self-prioritisation effect.

\subsection{General Methods}

The following section outlines the methods for all three studies, which utilise the same paradigm. Specifics to Experiment 1 are included thereafter.

\subsubsection{Participants}

All participants were recruited online via Prolific (www.prolific.ac) as native speakers of English with no hearing difficulties, and tested online using Gorilla (gorilla.sc, AnwylIrvine, Massonnié, Flitton, Kirkham, \& Evershed, 2018). Participants were also required to pass a headphone check (Woods, Siegel, Traer, \& McDermott, 2017) to ensure they were wearing headphones and able to hear the stimuli. 
None of the participants had taken part in any of the pilot studies associated with this project and, upon completion of the study, were paid for their participation. Ethical approval was obtained (UK) and informed consent was obtained from all participants prior to testing.

In Experiment 1, we recruited 35 participants (mean age $=27.25$ years, $S D=6.41$ years, age range $=18-40,29$ male). The sample size was determined according to a previous study using the same paradigm by Schäfer et al. (2016). Their a priori power calculation suggested an effect size of $d z \geq .52$ could be achieved given $N=31, \alpha=$ .05 , and a power of $1-\beta=.80$. A true effect size of $d z=.51$ was subsequently found. After exclusion criteria were applied (see 2.2.5 Pre-processing), data from 31 participants were analysed in Experiment 1.

\subsubsection{Stimuli}

Across all experiments, the stimuli included auditory exemplars of three male speakers and three female speakers each saying 'hello' in Southern Standard British English. The male-voiced exemplars were extracted from a larger pool of original recordings, which were obtained in sound-attenuated booths using desktop computers running Audacity (https://www.audacityteam.org/; RRID = SCR_007198) and either a Røde NT1A microphone (Røde Microphones, Sydney, Australia) or a Neumann TLM103 microphone (Neumann, Berlin, Germany). Ethical approval for these recordings was obtained (approval number 897). The female-voiced exemplars were recorded using a Rode NT-1A microphone via an RME Fireface UC audio interface. Recordings were digitised using Audacity (Audacity Team, 2019) as 16-bit, stereo WAV files, with sample rate $44100 \mathrm{~Hz}$. All stimuli were normalised for RMS amplitude using PRAAT (Boersma \& Weenink, 2010).

The three male voices and three female voices were each selected from a wider pool of voices based on trait ratings obtained in pilot studies. In the first pilot study, a group of listeners $(n=15$ participants, mean age $=30.33$ years, $S D=6.629$, age range $=$ 18-40 years, 8 female) rated 30 auditory exemplars of 'hello' from six different male speakers for attractiveness ('How attractive does this voice sound?') and, separately, for trustworthiness ('How trustworthy does this voice sound?') on a 7-point Likert scale 
whereby 1 denoted 'not attractive/trustworthy at all' and 7 denoted 'very attractive/trustworthy'. We selected three male voices for use in these experiments based on their being well matched in attractiveness (mean ratings \pm SDs $=4.6 \pm 1.2$, $4.5 \pm 1.3,4.8 \pm 1.4$ ) and trustworthiness (mean ratings \pm SDs $=4.6 \pm 1.2,5.2 \pm 1.1$, $4.7 \pm 1.2)$.

In the same pilot, participants were asked to rank the six different male voices in order of preference, 1 to 6 , whereby 1 denoted 'The voice I would most like to have as my own' down to 6, which denoted 'The voice I would least like to have as my own'. A Friedman Test was then run in the R environment using stats (R Core Team, 2013) to determine whether the six voices differed significantly in their mean ranking. The results indicated that the voices did differ significantly $(X 2(5)=23.82, p<.001)$ so further Nemenyi post-hoc tests were run using PMCMR (Pohlert, 2014). The comparisons revealed that, of the original six voices, the three male voices used across these three experiments were well matched in their mean ranking with all $p$ values

In a second pilot study, six female voices were also trait rated and ranked for preference in the same way $(n=15$ participants, mean age $=27.6$ years, $S D=6.31$, age range $=18-39$ years, 6 female). As with the male voice stimuli, the three female voices selected for use in Experiments 2 and 3 were chosen for being well matched for attractiveness (mean ratings \pm SDs $=4.4 \pm 1.0,4.2 \pm 1.4,4.8 \pm 1.4$ ) and for trustworthiness (mean ratings $\pm \mathrm{SDs}=4.8 \pm 1.2,4.6 \pm 1.3,4.9 \pm 1.0$ ).

Finally, the same participants were asked to rank the six different female voices in order of preference. The ranking data was analysed with a Friedman Test in the $\mathrm{R}$ environment and the results indicated that the voices differed significantly $(x 2(5)=$ 28.83, $\mathrm{p}<.001)$. Further Nemenyi post-hoc tests were run and the comparisons revealed that, of the original six voices, the three female voices used in Experiments 2 and 3 were well matched in their mean ranking with all p-values >.05.

Experiment 1 used just the male voice stimuli, with two auditory tokens of "hello" per voice. 


\subsubsection{Procedure}

The paradigm involved two stages: the familiarisation phase and the test phase. The online tasks here described can be accessed and previewed online via Gorilla Open Materials at: https://gorilla.sc/openmaterials/45935.

\section{1) Familiarisation Stage}

In the familiarisation stage, participants were passively exposed to each of the three voices and their associated identity label. Following a $500 \mathrm{~ms}$ fixation cross, the word 'you', 'friend', or 'stranger' was displayed in the centre of the screen in black uppercase font on a white background and remained onscreen for $3000 \mathrm{~ms}$. $500 \mathrm{~ms}$ after the label's onset, an auditory exemplar of 'hello' from the to-be-associated voice was played, lasting approximately 500ms. After the auditory stimulus finished, the label remained on the screen until the end of the $3000 \mathrm{~ms}$ trial. The familiarisation phase consisted of 12 trials, with each label-voice pairing being presented four times, and each of the two auditory exemplars per voice played twice. Stimuli were presented in a random order. The familiarisation stage thus lasted approximately 1 minute, including on-screen instructions, and was immediately followed by the test phase.

\section{2) Test Phase}

The test phase started with 12 practice trials. Each trial started with a 500ms fixation cross in the centre of the screen, after which an auditory exemplar played for its total duration (approx. 500ms). Immediately after the auditory offset, the word 'you', 'friend', or 'stranger' was displayed in the centre of the screen. Participants were asked to judge whether the identity label onscreen was a match or mismatch to the voice heard and to respond by keyboard press as quickly and as accurately as possible. Participants were instructed to press the left arrow for 'MATCH' and the right arrow for 'MISMATCH'. This left-right ordering of match and mismatch remained constant on all trials, for all participants. In the practice trials only, text appeared on-screen to remind participants that a left button press meant a match, and a right button press meant mismatch. In all test trials, however, no visual reminder was provided. Feedback was given onscreen for $500 \mathrm{~ms}$ immediately following every response: a green tick for correct, a red cross for incorrect, and text feedback of 'TOO SLOW' for responses 
occurring after $1500 \mathrm{~ms}$. The next trial began after the $500 \mathrm{~ms}$ feedback period was complete.

Following the practice trials, participants performed three blocks of 72 trials (216 trials in total) and were informed of their overall accuracy at the end of each block. The order of match vs. mismatch trials was randomised for all participants. The experiment lasted approximately 25 minutes in total and participants were debriefed at the end.

\subsubsection{Design}

Experiment 1 consisted of a within-subjects factor, 'voice identity' (self vs. friend vs. stranger voice) $\times$ a within-subjects factor, 'trial type' (match vs. mismatch) design.

\subsubsection{Pre-processing}

In each experiment we analysed three measures: reaction times, sensitivity, and accuracy. The first two measures were included to align with previous reports of the self-prioritisation effect by Sui et al. (2012) and Schäfer et al. (2016). We also included trialwise accuracy to allow us to run a complementary analysis to the RT mixed model that included the same random effects structure.

Reaction times: RTs were measured as the delay between the onset of the visual label and the participant's response and categorised by the voice identity (self-voice, friendvoice, stranger-voice) per trial, and not by the category of the presented label ("YOU", "FRIEND", "STRANGER"). All erroneous responses, as well as responses shorter than 200 ms or longer than 1500 ms were excluded from the RT analysis in line with Sui et al.'s (2012) approach.

Sensitivity: unbiased d' scores were calculated by combining performance scores from match and mismatch trials at each level of the voice identity factor (self, friend, stranger). Specifically, YES/NO responses were recoded into hits and false alarms, following the log-linear approach to adjust for cases involving $100 \%$ hits or $0 \%$ false alarms (Stanislaw \& Todorov, 1999). 
Accuracy: Trialwise accuracy scores were recorded for each participant. Whole datasets for participants showing overall performance accuracy at chance $(\leq 50 \%+$ $95 \% \mathrm{Cl}$ ) were excluded as this indicated random responses.

In Experiment 1, 2.48\% of the trials were eliminated on the RT criteria above as well as whole datasets for two participants with more than $50 \%$ of responses shorter than $200 \mathrm{~ms}$. A further two datasets were eliminated on the accuracy criterion. None of these participants was replaced, therefore data from a total of 31 participants were analysed in Experiment 1.

\subsubsection{Analysis}

Across all experiments, reaction times and sensitivity were assessed with linear mixed models (LMM) using Ime4 (Bates, Maechler, Bolker \& Walker, 2014) in the $R$ environment ( $R$ Core Team, 2013). Accuracy was assessed using binomial generalised linear mixed models (GLMM). In every analysis (with the exception of sensitivity in Experiment 1), we ran a model that included an interaction between voice identity and the manipulated variable, two fixed effects (voice identity, and the manipulated variable: trial type in Experiment 1, gender-matching in Experiment 2, and choice in Experiment 3), and a random intercept of 'participant'. Statistical significance of the effects was established via likelihood ratio tests by dropping effects of interest from the appropriate model. For example, to establish whether the interaction was significant, we dropped this interaction from the model including both fixed effects. To test for the significance of either of the main effects, we dropped the relevant effects from a model that only included the two main effects. For all analyses reported here, post-hoc comparisons were conducted in emmeans (Lenth, 2016) and were adjusted for the multiple comparisons via Bonferroni correction.

For GLMMs, odds ratios and associated confidence intervals are provided as an estimate of the size of the relevant effects. An odds ratio of 1 indicates that no effect is present, while odds ratios that deviate from 1 indicate an effect is present. The larger the deviation, the bigger the effect. If the confidence intervals do not cross 1 , the relevant effect is significant. For LMMs, the models' estimates and associated confidence intervals for each effect are reported. The further away from 0 the 
estimates are, the bigger the effect. If the confidence intervals do not cross 0 , the relevant effect is significant.

In addition to the linear mixed models, we also ran ANOVAs on all three measures to enable a more direct comparison with previous published studies. The results can be found in Supplemental Materials.

\subsection{Results \& Discussion}

Descriptive statistics for reaction times, accuracy, and sensitivity scores (d') for the different conditions and voice identities are given in Table 1.

Table 1. Mean RTs, accuracy, and d-primes for Experiment 1.

\begin{tabular}{|c|c|c|c|c|}
\hline Condition & Voice Identity & $\begin{array}{l}\text { Mean } \\
\text { (ms) }\end{array}$ & Accuracy & $d^{\prime *}$ \\
\hline \multirow[t]{3}{*}{ Match trials } & Self & $584(211)$ & $0.91(0.29)$ & $2.64(1.11)$ \\
\hline & Friend & $635(220)$ & $0.88(0.33)$ & $2.31(0.97)$ \\
\hline & Other & $645(227)$ & $0.87(0.34)$ & $2.29(1.06)$ \\
\hline \multirow[t]{3}{*}{ Mismatch trials } & Self & $714(242)$ & $0.83(0.38)$ & \\
\hline & Friend & $722(217)$ & $0.81(0.39)$ & \\
\hline & Other & $712(216)$ & $0.81(0.40)$ & \\
\hline
\end{tabular}

Note. $\mathrm{RT}$ = reaction time; Accuracy = proportion correct. Standard deviations appear within parentheses. *Performance scores from match and mismatch trials are combined to provide d' scores.

\subsubsection{Reaction times}

The reaction time data are plotted in Figure 1. We ran an LMM on RTs to assess whether the self-voice had been perceptually prioritised relative to the others depending on the trial type (match trials vs mismatch trials). For the post-hoc tests, alpha was set as .008 after Bonferroni correction for six comparisons.

$$
\begin{aligned}
& \text { Imer(reaction time } \sim \text { voice identity * trial type } \\
& \quad+(1 \text { participant }), \text { REML = "FALSE") }
\end{aligned}
$$


Table 2. Model output for the analysis of the reaction times in Experiment 1.

The reference category for Voice Identity is "Self"; the reference category for Trial Type is "Match".

(Intercept)

Main Effect Voice Identity

Voice identity (Friend)

Voice identity (Other)

Main Effect Trial Type

Trial Type (Mismatch)

Interaction Voice Identity * Trial Type

Voice identity (Friend) * Trial Type

(Mismatch)

Voice identity (Other) * Trial Type (Mismatch)
Estimates

581.29

53.57

62.22

132.12

$-62.65$
$\mathrm{Cl}$

$555.40-607.18$

$34.67-72.48$

$43.19-81.24$

$112.87-151.37$

$-72.72--17.97$

$-90.18--35.12$

Full model outputs are reproduced in Table 2. There was a significant interaction between voice identity and trial type $\left(X^{2}(2)=21.32, p<.001\right)$. Post-hoc tests confirmed that reaction times for the self-voice were significantly quicker than those for the friendvoice $(p<.001)$ and the stranger-voice $(p<.001)$. However, this was only the case within matched trials, which is in line with previous studies. There was no significant difference between reaction times for the friend-voice and stranger-voice in either match, or mismatch trials (ps > .008). These results show therefore a significant selfprioritisation effect for the perception of voices, thus supporting our hypothesis. 


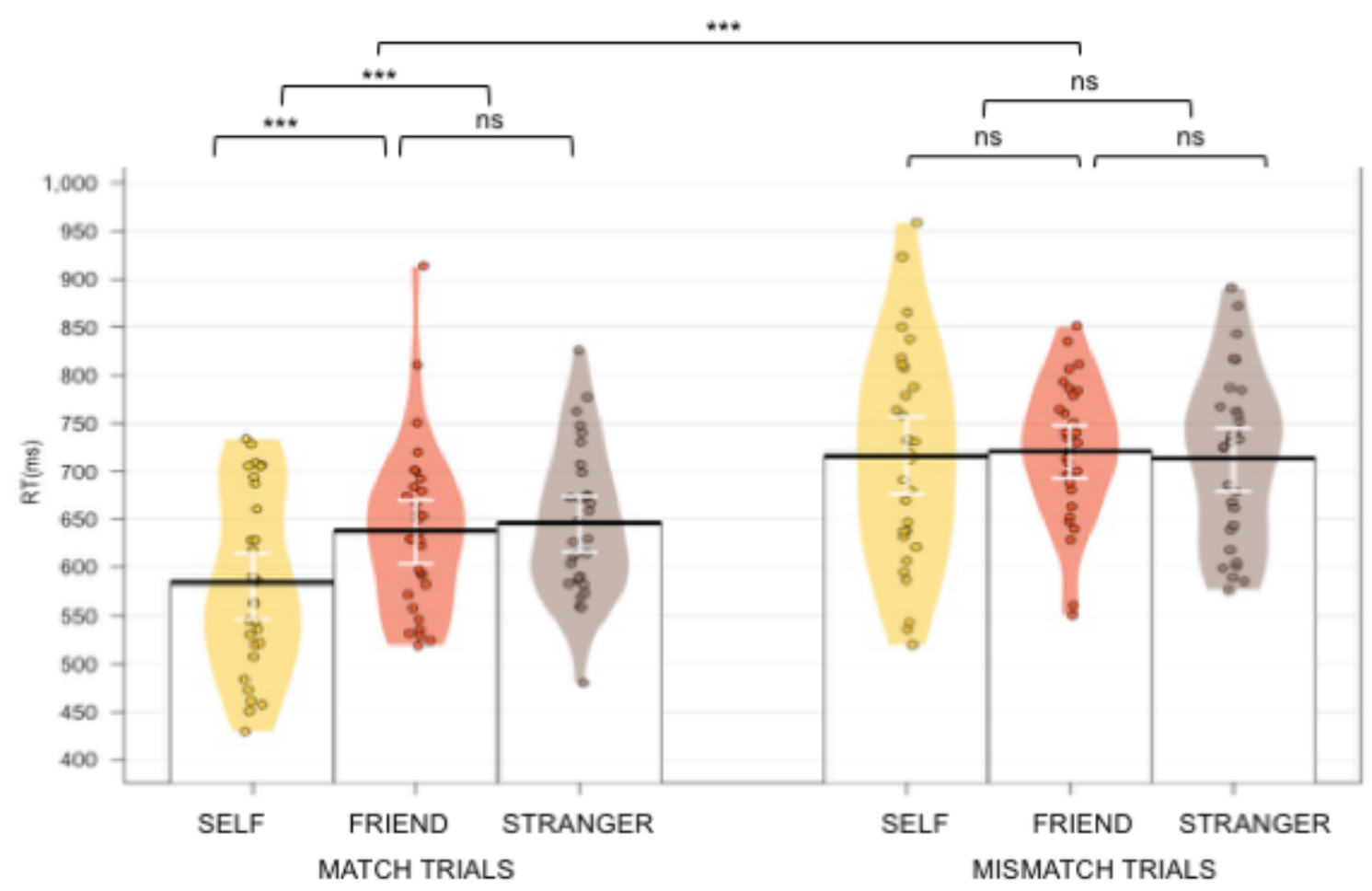

Figure 1. Experiment 1: Mean reaction times (RT) as a function of the voice identity (self, friend, or stranger) and the trial type (match vs mismatch). The error bars indicate the SEs of the means. Coloured segments show smoothed density curves for the full data distribution, while individual dots indicate mean RTs per participant. Horizontal bars show post-hoc comparisons with asterisks denoting significance within RTs as determined via likelihood ratio tests (see Results). ${ }^{* * *} p<.001$.

\subsubsection{Sensitivity}

We further assessed the self-prioritisation effect by running an LMM on d' scores to assess performance using the model below. For the post-hoc tests, alpha was set as .017 after Bonferroni correction for three post-hoc comparisons.

$$
\text { Imer (sensitivity voice identity + (1|participant), REML = "FALSE") }
$$

Table 3. Model output for the analysis of the reaction times in Experiment 1. The reference category for Voice Identity is "Self".

$\begin{array}{lll}\text { (Intercept) } & 2.62 & 2.25-2.98 \\ \begin{array}{l}\text { Main Effect Voice } \\ \text { Identity }\end{array} & & \\ \quad \text { Voice identity (Friend) } & -0.33 & -0.64--0.02 \\ \quad \text { Voice identity (Other) } & -0.36 & -0.67--0.06\end{array}$


Full model outputs are reproduced in Table 3 . There was again a significant main effect of voice identity $\left(x^{2}(2)=6.24, p=.044\right)$. However, post hoc comparisons showed that although sensitivity for the self-voice was numerically greater than that for both the friend-voice and stranger-voice, the difference did not reach significance (ps $>$.017) after Bonferroni correction.

\subsubsection{Accuracy}

We also ran a GLMM on trialwise accuracy. For the post-hoc tests, alpha was set as .017 after Bonferroni correction for three post-hoc comparisons.

$$
\begin{aligned}
& \text { glmer(accuracy } \sim \text { voice identity * trial type } \\
& +(1 \text { |participant }), \text { family }=\text { "binomial" })
\end{aligned}
$$

Table 4. Model output for the analysis of accuracy in Experiment 1.

The reference category for Voice Identity is "Self", the reference category for Trial Type is "Match".

Odds

Ratios $\mathrm{Cl}$

(Intercept)

12.85

$8.98-18.38$

Main Effect Voice Identity

Voice identity (Friend)

$0.7 \quad 0.53-0.93$

Voice identity (Other)

$0.64 \quad 0.48-0.84$

Main Effect Trial Type

Trial Type (Mismatch)

Interaction Voice Identity * Trial Type

$0.46 \quad 0.35-0.60$

Voice identity (Friend) * Trial Type

(Mismatch)

$1.25 \quad 0.87-1.80$

Voice identity (Other) * Trial Type (Mismatch)

$1.3 \quad 0.91-1.86$

Full model outputs are reproduced in Table 4. The interaction of voice identity and trial type was not significant $\left(X^{2}(2)=2.29, p=.318\right)$. There was, however, a significant main effect of voice identity $\left(x^{2}(2)=11.84, p=.002\right)$, showing that performance accuracy for the self-voice was higher than for both the friend-voice $(p=.013)$ and the stranger-voice $(p<.001)$, while the friend-voice and stranger-voice did not significantly differ. There was also a main effect of trial type $\left(x^{2}(1)=70.63, p<.001\right)$ showing that accuracy was 
significantly higher in match trials than in mismatch trials $(p<.001)$; a result which falls in line with previous studies.

In Experiment 1, we asked whether an unfamiliar voice could become self-associated and measured the processing bias subsequently afforded to this new self-voice relative to voices newly associated with a friend or stranger. Our results showed a clear self-prioritisation effect, with quicker reaction times, higher accuracy, and increased perceptual sensitivity to the self-voice relative to other voice identities. This therefore supports our hypothesis and provides a pattern of results that mirrors those reported by Sui et al. (2012), Schafer et al. (2016), and Payne et al. (2017). This demonstrates for the first time that our 'self' can quickly be extended to incorporate an unfamiliar voice - that inherently belongs to another - as our own. We further show that voices do not need to be self-generated in order to be self-associated and perceptually prioritised as a self-relevant signal. This has positive implications for the potential uptake of synthesised voices by patients with vocal impairments.

\section{Experiment 2}

Having demonstrated that there is a self-prioritisation effect in the perception of voices, Experiment 2 asked whether this effect is increased if the new self-voice is gendermatched to the identity of the listener. This experiment was preregistered via the Open Science Framework (https://osf.io/r6g $2 \mathrm{~m} /$ ) and can be previewed via Gorilla Open Materials at: https://gorilla.sc/openmaterials/46084.

People prefer others whom they perceive or imagine to be similar to themselves because it activates the positive and implicit associations they already hold about themselves (Jones, Pelham, Carvallo, \& Mirenberg, 2004). This perceived similarity reduces the social distance between them and, relatedly, the degree to which the self and the other are differentially processed (Benoit, Gilbert, Volle, \& Burgess, 2010). For instance, people are able to recall objects belonging to a similar other better than objects belonging to a dissimilar other (Allan, Morson, Dixon, Martin, and Cunningham, 2017). This shows that the perceived closeness of an object's association to the self can influence the way we process it: more closely associated items are more prioritised in memory. 
It is reasonable therefore to suggest that an other-generated voice that is more similar to the true self-voice of the listener - in terms of gender-related acoustics - may be perceived as more closely related to the self than a voice that represents a less similar other. Voice characteristics arise from, and subsequently convey, information about our physical self-identity including body size, shape, health and gender. In adult humans, sexual dimorphism in voice characteristics - including fundamental frequency and formant spacing - is extremely large in comparison with other species (Puts et al., 2016). Further, the differences in these voice characteristics are perceptually highly salient (Lass et al., 1976; Owren, Berkowitz \& Bachorowski, 2007). Thus, through manipulating the sex of the experimental voice identities, and their match to the gender identity of participants, we can effectively test whether an externally-generated vocal signal that better fits within the parameters of the participant's vocal apparatus and self-identity may be deemed more personally relevant than a voice that signals a different gender identity.

\subsection{Hypothesis}

We hypothesised that the self-prioritisation effect for the self-voice will increase when it is matched to the gender identity of the listener. Such an increase should result in quicker reaction times and higher accuracy in response to the self-voice, relative to the friend- or stranger-voice, when the self-voice is matched to the gender identity of the listener as compared to when the self-voice is not matched to the gender identity of the listener.

\subsection{Methods}

Unless stated below, the methods for Experiment 2 replicated methods from Experiment 1.

\subsubsection{Participants}

96 participants took part ( 48 females: mean age $=29.1$ years, $S D=6.28$ years, age range $=18-40 ; 48$ males: mean age $=27.4$ years, $S D=6.58$ years, age range $=18$ 40). The data from participants $(\mathrm{N}=2)$ showing overall performance accuracy at chance $(\leq 50 \%+95 \% \mathrm{Cl})$ were excluded and replacement participants sampled such that data from 96 participants were included in the reported analyses. 


\subsubsection{Randomisation}

Male and female participants were randomly allocated to either a 'gender-matched' group ( $N=48: 24 \mathrm{M}, 24 \mathrm{~F}$ ) or a 'non-gender-matched' group ( $N=48: 24 \mathrm{M}, 24 \mathrm{~F}$ ), whereby the gender of the participant would match or not match the gender of the voices.

\subsubsection{Stimuli}

In addition to the male voice stimuli used in Experiment 1, we included female voice stimuli from three SSBE-accented speakers.

\subsubsection{Design}

The study consisted of a within-subjects factor, 'voice identity' (self vs. friend vs. stranger) $\times$ a between-subjects factor, 'gender-matching' (gender-matched vs nongender-matched) design. Further, a within-subjects factor of trial type was present (match vs mismatch trials).

\subsubsection{Pre-processing}

Based on the exclusion criteria reported earlier, fewer than $1 \%$ of the trials were eliminated.

\subsubsection{Analysis}

In Experiment 1 we found that the self-prioritisation effect emerged on match trials only (i.e. trials in which the presented voice and identity label are correctly 'matched' according to their learnt association). Therefore, following the logic of Sui et al. (2012) and Schafer et al. (2016), the analyses of reaction times and raw accuracy are henceforth reported separately for match and mismatch trials.

\subsection{Results \& Discussion}

Descriptive statistics for reaction times, accuracy, and sensitivity scores (d') for the different conditions and voice identities are given in Table 5. 
Table 5. Mean RTs, accuracy and d-prime scores in matched trials for Experiment 2.

\begin{tabular}{lllll}
\hline Condition & Voice Identity & Mean RT $(\mathbf{m s})$ & Accuracy & $\boldsymbol{d}^{\mathbf{}^{*}}$ \\
\hline Gender-matched & Self & $541(203)$ & $0.92(0.27)$ & $2.89(0.98)$ \\
& Friend & $572(213)$ & $0.92(0.27)$ & $2.90(1.01)$ \\
& Other & $592(221)$ & $0.85(0.35)$ & $2.49(0.97)$ \\
Non-gender- & Self & $536(205)$ & $0.94(0.24)$ & $2.95(1.04)$ \\
matched & & $570(222)$ & $0.91(0.29)$ & $2.87(1.16)$ \\
& Friend & $589(224)$ & $0.88(0.33)$ & $2.68(1.21)$ \\
\hline
\end{tabular}

Note. RT = reaction time; Accuracy - proportion correct. Standard deviations appear within parentheses. *Performance scores from match and mismatch trials are combined to provide d'scores.

\subsubsection{Reaction times}

The reaction time data is plotted in Figure 2. As with analysis in Experiment 1, we ran an LMM on RTs to assess whether the self-voice had been perceptually prioritised. First, we analysed match trials only. For the post-hoc tests, alpha was set as .017 after Bonferroni correction for three post-hoc comparisons.

$$
\begin{gathered}
\text { Imer(Reaction time } \sim \text { voice identity * gender-matching } \\
+(1 \text { participant }), \text { REML }=\text { "FALSE" })
\end{gathered}
$$

Table 6. Model output for the analysis of the reaction times in Experiment 2.

The reference category for Voice Identity is "Self", the reference category for Gender Matching is "No".

$\begin{array}{lrr} & \text { Estimates } & \mathrm{Cl} \\ \text { (Intercept) } & 540.52 & 514.21-566.83 \\ \text { Main Effect Voice Identity } & & \\ \quad \text { Voice identity (Friend) } & 35.35 & 21.58-49.12 \\ \quad \text { Voice identity (Other) } & 52.2 & 38.30-66.10 \\ \text { Main Effect Gender Matching } & & \\ \quad \text { Gender Matching (Yes) } & 0.68 & -36.55-37.91 \\ \text { Interaction Voice Identity * Gender Matching } & & \\ \quad \text { Voice identity (Friend) * Gender Matching (Yes) } & -5.02 & -24.49-14.46 \\ \quad \text { Voice identity (Other) * Gender Matching (Yes) } & -1.5 & -21.29-18.28\end{array}$


Full model outputs are reproduced in Table 6 . The interaction of voice identity and gender-matching was non-significant $\left(x^{2}(2)=.27, p=.875\right)$. There was, however, again a main effect of voice identity $\left(X^{2}(2)=106.78, p=.001\right)$ : In both groups, reaction times for the self-voice were significantly quicker than those for either the friend-voice $(p<.001)$ or the stranger-voice $(p<.001)$, and reaction times to the friend voice were also significantly quicker than those for the stranger-voice $(p<.001)$. There was no effect of gender matching on reactions times $\left(x^{2}(1)<.01, p=.934\right)$.

These RT data therefore reveal a significant self-prioritisation effect in the perception of voices, but show that prioritisation was not modulated according to whether the selfvoice was matched to the gender identity of the listener.

\section{$\underline{\text { Match Trials }}$}

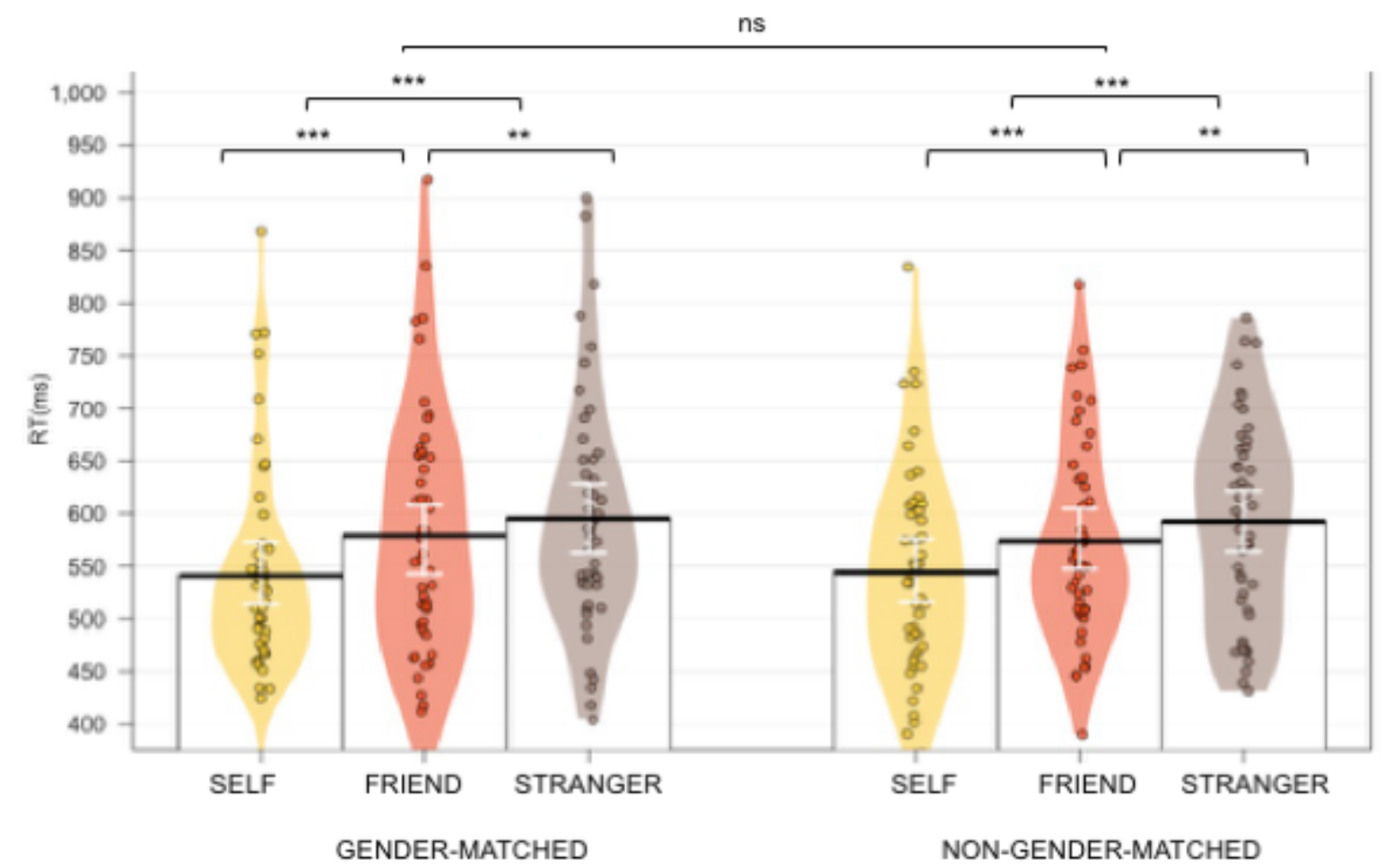

Figure 2. Experiment 2: Mean reaction times (RT) as a function of the voice identity (self, friend, or stranger) and gender-matching (matched vs non-matched i.e. whether the voice matches the gender identity of the listener, or not). The effect of gender-matching was not significant. Graph models match trials only. The error bars indicate the SEs of the means. Coloured segments show smoothed density curves for the full data distribution, while individual dots indicate mean RTs per participant. Horizontal bars show post-hoc comparisons with asterisks denoting significance within RTs as determined via likelihood ratio tests (see Results). ${ }^{* *} p<.001$. 
Identical analyses of RT data were then run on the mismatch trials, but the interaction effect $(x 2(2)=1.03, p=.596)$, the effect of voice identity, $(x 2(2)=.07, p=.968)$, and the effect of gender matching $(x 2(2)=.69, p=.407)$ were all non-significant in mismatch trials.

\subsubsection{Sensitivity}

We also ran an LMM on d' scores. For the post-hoc tests, alpha was set as .017 after Bonferroni correction for three post-hoc comparisons.

$$
\begin{gathered}
\text { Imer(sensitivity voice identity * gender-matching } \\
+(1 \text { participant), REML = "FALSE") }
\end{gathered}
$$

Table 7. Model output for the analysis of sensitivity in Experiment 2.

The reference category for Voice Identity is "Self", the reference category for Gender Matching is "No".

$\begin{array}{lrr} & \text { Estimates } & \mathrm{Cl} \\ \text { (Intercept) } & 2.95 & 2.66-3.25 \\ \text { Main Effect Voice Identity } & & \\ \quad \text { Voice identity (Friend) } & -0.08 & -0.33-0.16 \\ \quad \text { Voice identity (Other) } & -0.28 & -0.52--0.03 \\ \text { Main Effect Gender Matching } & & \\ \quad \text { Gender Matching (Yes) } & -0.07 & -0.49-0.36 \\ \quad \text { Interaction Voice Identity * Gender Matching } & & \\ \quad \text { Voice identity (Friend) * Gender Matching (Yes) } & 0.1 & -0.25-0.45 \\ \quad \text { Voice identity (Other) * Gender Matching (Yes) } & -0.12 & -0.47-0.22\end{array}$

Full model outputs are reproduced in Table 7 . The interaction of voice identity and gender-matching was non-significant $(X 2(2)=1.57, p=.455)$. There was again a main effect of voice identity $(x 2(2)=16.62, p<.001)$, with post-hoc comparisons revealing that, across groups, d-primes for the self-voice were significantly higher than those for the stranger-voice $(p<.001)$ but did not differ significantly from the friend-voice. Further, $d$-primes for the friend-voice were significantly higher than those for strangervoice $(p<.001)$. There was again no effect of gender-matching on reaction times $(x 2(1)=.16, p=.687)$. 


\subsubsection{Accuracy}

Finally, we ran a GLMM on trialwise accuracy. Here we analysed the match trials. For the post-hoc tests, alpha was set as .008 after Bonferroni correction for six comparisons.

$$
\begin{gathered}
\text { glmer(accuracy } \sim \text { voice identity * gender-matching } \\
+(1 \mid \text { participant }), \text { family = "binomial" })
\end{gathered}
$$

Table 8. Model output for the analysis of accuracy in Experiment 2.

The reference category for Voice Identity is "Self", the reference category for Gender Matching is "No".

Odd Ratio

$$
\text { (Intercept) }
$$

Main Effect Voice Identity

Voice identity (Friend)

Voice identity (Other)

Main Effect Gender Matching

Gender Matching (Yes)

Interaction Voice Identity * Gender Matching

Voice identity (Friend) * Gender Matching (Yes)

Voice identity (Other) ${ }^{*}$ Gender Matching (Yes)
9.2

1.08

1

0.94

0.98
$\mathrm{Cl}$

$6.52-12.98$

$0.90-1.36$

$0.88-1.33$

$0.61-1.61$

$0.70-1.26$

$0.73-1.32$

Full model outputs are reproduced in Table 8. There was a significant interaction between voice identity and gender-matching on accuracy $\left(x^{2}(2)=7.09, p=.029\right)$. Post hoc comparisons revealed that accuracy for the self-voice was significantly higher than accuracy for the stranger-voice $(p<.001)$ in both gender-matched and non-gendermatched groups. However, accuracy for the self-voice only differed from the friendvoice in the non-gender-matched group $(p=.001)$. Accuracy for the self-voice did not differ significantly when it was gender-matched in comparison to when it was not.

Identical analyses within the mismatch trials revealed that neither the interaction effect $\left(x^{2}(2)=1.03, p=.596\right)$, the fixed effect of voice identity $\left(x^{2}(2)=.065, p=.968\right)$, nor the fixed effect of gender-matching $\left(X^{2}(1)=.069, p=.408\right)$ were significant.

In Experiment 2, we asked whether the self-prioritisation effect could be modulated by the self-voice being gender-matched to the identity of the listener. Our results show 
that gender-matching did not significantly influence prioritisation here, with equivalent reaction times and perceptual sensitivity to the voices when they were matched as to when they were mismatched. Although we observed a significant interaction of voice identity and gender-matching for accuracy scores, this did not manifest in interpretable differences in terms of how the self-voice related to the friend and stranger voices across the two groups. Overall, these results suggest that the perceived physiological similarity between the novel voices and the participant's own voice has no effect on the self-prioritisation effect. This is unexpected (see the General Discussion for suggested explanations). Instead, prioritisation was similar for both groups, in that perceptual processing was biased towards the new self-voice relative to the friend- or stranger-voice, thereby supporting the results from Experiment 1.

\section{Experiment 3}

Experiment 3 further investigated which factors may influence the acceptability of an externally-generated voice being integrated into self-representation. Specifically, we asked whether the action of choosing a new voice for the self increases the level of bias towards it, and the implications of this on the prioritisation effect. This experiment was preregistered via the Open Science Framework (https://osf.io/wz2am/) and can be previewed via Gorilla Open Materials at: https://gorilla.sc/openmaterials/46086.

The effect of choice on self-bias has been investigated in the memory domain by Cloutier and Macrae (2008). They demonstrate that participants who are given a choice over which items to own can better recall those items relative to participants who had no choice and were instead afforded ownership by someone else. The act of selection, of making a personal choice over external stimuli, is associated with enhanced memory for the later outcomes of those choices (Cloutier \& Macrae, 2008). Further, self-selected items are more likely to align with the current preferences and biases of the self, and so be more strongly encoded to the self - and more prioritised - than unchosen items.

The extent to which the amount of personal choice we have influences our degree of bias has also been explored by Cunningham et al. (2008; 2011). They demonstrate that bias towards an item only occurs when it is both 'self-owned' and 'self-chosen', showing that having more personal choice increases bias. This is mirrored in Huang, 
Wang, and Shi's (2009) study, in which the bias that was afforded by merely selfowning an item (relative to an other-owned item) disappeared when participants are given a chance to compare their own items with another's. In contrast, the bias afforded by self-choosing an item, either to become a self-owned item or to give as an other-owned item, remained stable regardless of the ownership. Thus, encoding for a self-chosen item seems to be stronger and able to override ownership biases.

\subsection{Hypothesis}

Experiment 3 therefore predicts that the self-prioritisation effect in voices will be increased if the new, self-voice is first chosen by the listener, relative to when it has been randomly allocated. Such an increase in prioritisation should result in quicker reaction times and higher accuracy in response to the self-voice - in comparison to the voices associated with the friend or stranger - when the new self-voice has been chosen, relative to when it has been randomly allocated.

\subsection{Methods}

Unless stated below, the methods for Experiment 3 replicated methods from Experiment 1 and 2.

\subsubsection{Participants}

96 new participants were recruited, forming a 'choice' group (48 females: mean age $=$ 29.2 years, $S D=6.27$ years, age range $=18-40 ; 48$ males: mean age $=26.5$ years, $\mathrm{SD}=6.71$ years, age range $=18-40$ ). As in previous analysis, datasets for participants showing overall performance accuracy at chance $(\leq 50 \%+95 \% \mathrm{Cl})$ were excluded $(\mathrm{N}=3)$, as this indicated random responses. These participants were replaced to reach the full sample size of 96 participants. Data from these 96 participants were then combined with data from the 96 participants from Experiment 2 who had been randomly allocated voices, thus forming the 'no choice' group (48 females: mean age $=29.1$ years, $S D=6.28$ years, age range $=18-40 ; 48$ males: mean age $=27.4$ years, $S D=6.58$ years, age range $=18-40)$. Thus, data from 192 participants were included in this study.

\subsubsection{Stimuli}


Experiment 3 used both male and female voice stimuli, as in Experiment 2.

\subsubsection{Design}

The study consisted of a factorial design with one within-subjects factor, 'voice identity' (self vs. friend vs. stranger) and one between-subjects factor, 'choice' (choice vs. no choice). Further, a within-subjects factor of trial type was present (match vs mismatch trials).

\subsubsection{Procedure}

For the 'choice' group, Experiment 3 began with a voice selection task in which the participants were presented with two tokens of 'hello' from each of the three male voices, labelled onscreen as 'Voice A', 'Voice B', and 'Voice C'. They were then asked to choose which voice they wanted to represent them for the rest of the study. The voices for 'friend' and 'stranger' were allocated randomly, and evenly, thereafter. Second, participants were asked to rate the strength of their preference for their chosen voice on a 5-point scale where 1 denoted, 'Not strong at all: I was indifferent/picked randomly' and 5 denoted, 'Very strong: I preferred my chosen voice much more than the others.'

Thereafter, the procedure replicated that of Experiments 1 and 2.

\subsubsection{Pre-processing}

In the self-voice selection task, participants were presented with a choice of 3 male voices, or 3 female voices. For participants choosing from male voices, there was a relatively balanced selection across the three identities. Voice 1 was chosen by $37.5 \%$ of participants (8F, 10M), Voice 2 was also chosen by $37.5 \%$ of participants $(8 \mathrm{~F}, 10 \mathrm{M})$, and Voice 3 was chosen by $25 \%$ of participants $(8 \mathrm{~F}, 4 \mathrm{M})$. The strength of preference task showed that $87.5 \%$ of participants stated they had a preference strength of at least three (out of five) for the voice they chose, with only $2.08 \%$ stating that they were indifferent to their self-voice.

Uptake of the female voices as the new self-voice was less balanced; Voice 1 was chosen by $62.5 \%$ of participants $(16 \mathrm{~F}, 15 \mathrm{M})$, Voice 2 was chosen by $12.5 \%(4 \mathrm{~F}, 2 \mathrm{M})$, 
and Voice 3 was chosen by $25 \%$ of participants (4F, 7M). The strength of preference task showed that $95.8 \%$ of participants stated they had a preference strength of at least three (out of five) for the voice they chose.

Since we posit that encoding of a stimulus to the self is strengthened through making a personal choice - rather than by the strength of preference for that stimulus - data from all participants were analysed regardless of their stated preference strength.

$1.18 \%$ of the trials were eliminated from the analysis RT based on our exclusion criteria.

\subsubsection{Analysis}

For the analyses of reaction times and raw accuracy, results within match and mismatch trials are reported separately, following the logic of Sui et al. (2012) and Schafer et al. (2016). Matched trials are those in which the presented voice and identity label are correctly 'matched' according to their learnt association.

\subsection{Results \& Discussion}

Descriptive statistics for reaction times, accuracy, and sensitivity scores (d') for the different conditions and voice identities are given in Table 9.

Table 9. Mean RTs, accuracy, and d-prime scores in matched trials for Experiment 3

\begin{tabular}{lllll}
\hline Condition & Voice Identity & Mean RT $(\mathbf{m s})$ & Accuracy & $\boldsymbol{d}^{\mathbf{}^{*}}$ \\
\hline Choice & Self & $542(204)$ & $0.94(0.25)$ & $3.10(1.06)$ \\
& Friend & $610(231)$ & $0.90(0.30)$ & $2.83(1.15)$ \\
No Choice & Other & $606(234)$ & $0.87(0.33)$ & $2.66(1.19)$ \\
& Self & $539(204)$ & $0.93(0.27)$ & $2.92(1.01)$ \\
& Friend & $571(218)$ & $0.92(0.28)$ & $2.89(1.09)$ \\
& Other & $590(222)$ & $086(0.34)$ & $2.59(1.10)$
\end{tabular}

Note. RT = reaction time; Accuracy - proportion correct. Standard deviations appear within parenthesis. *Performance scores from match and mismatch trials are combined to provide d' scores. 


\subsubsection{Reaction times}

The reaction time data is plotted in Figure 3 for the match trials and in Figure 4 for the mismatch trials. We ran an LMM on RT data to assess whether the self-voice had been perceptually prioritised relative to the others depending on choice (whether the self-voice was chosen or not). First, we analysed match trials only. For the post-hoc tests, alpha was set as .008 after Bonferroni correction for six comparisons.

$$
\begin{aligned}
& \text { Imer(reaction time } \sim \text { Voice identity * choice } \\
& \quad+(\text { 1 } \text { participant }), \text { REML = "FALSE" })
\end{aligned}
$$

Table 10. Model output for the analysis of the reaction times in Experiment 3. The reference category for Voice Identity is "Self", the reference category for Choice is "No".

$\begin{array}{lrr} & \text { Estimates } & \mathrm{Cl} \\ \text { (Intercept) } & 540.87 & 520.53-561.22 \\ \text { Main Effect Voice Identity } & & \\ \quad \text { Voice identity (Friend) } & 32.84 & 23.07-42.61 \\ \quad \text { Voice identity (Other) } & 51.44 & 41.52-61.37 \\ \text { Main Effect Choice } & & \\ \quad \text { Choice (Yes) } & 5.73 & -23.03-34.50 \\ \text { Interaction Voice Identity * Choice } & & \\ \quad \text { Voice identity (Friend) * Choice (Yes) } & 36.93 & 23.10-50.76 \\ \quad \text { Voice identity (Other) * Choice (Yes) } & 15.49 & 1.47-29.51\end{array}$

Full model outputs are reproduced in Table 10. There was an interaction between voice identity and choice $(X 2(2)=27.56,(p<.001)$, showing reaction times were quicker for the self-voice than for friend $(p<.001)$, or stranger $(p<.001)$ in both groups. We hypothesised that an interaction would arise because of a difference in RTs to the selfvoice when it is chosen in comparison to when it is not. However, instead, the interaction arose from a difference in RTs to the friend-voice when the self-voice was chosen in comparison to when it was not. Specifically, when the self-choice is chosen the RTs for the friend voice increase such that they become equivalent to those of the stranger's voice $(p=.577)$. This leaves the self-voice relatively more prioritised than either of the other two voices, which is not the case when the self-voice is randomly allocated. When the self-voice is randomly allocated, reaction times to friend and 
stranger $(p<.001)$ are significantly different such that there is less of a prioritisation of the self-voice relative to the other voices.

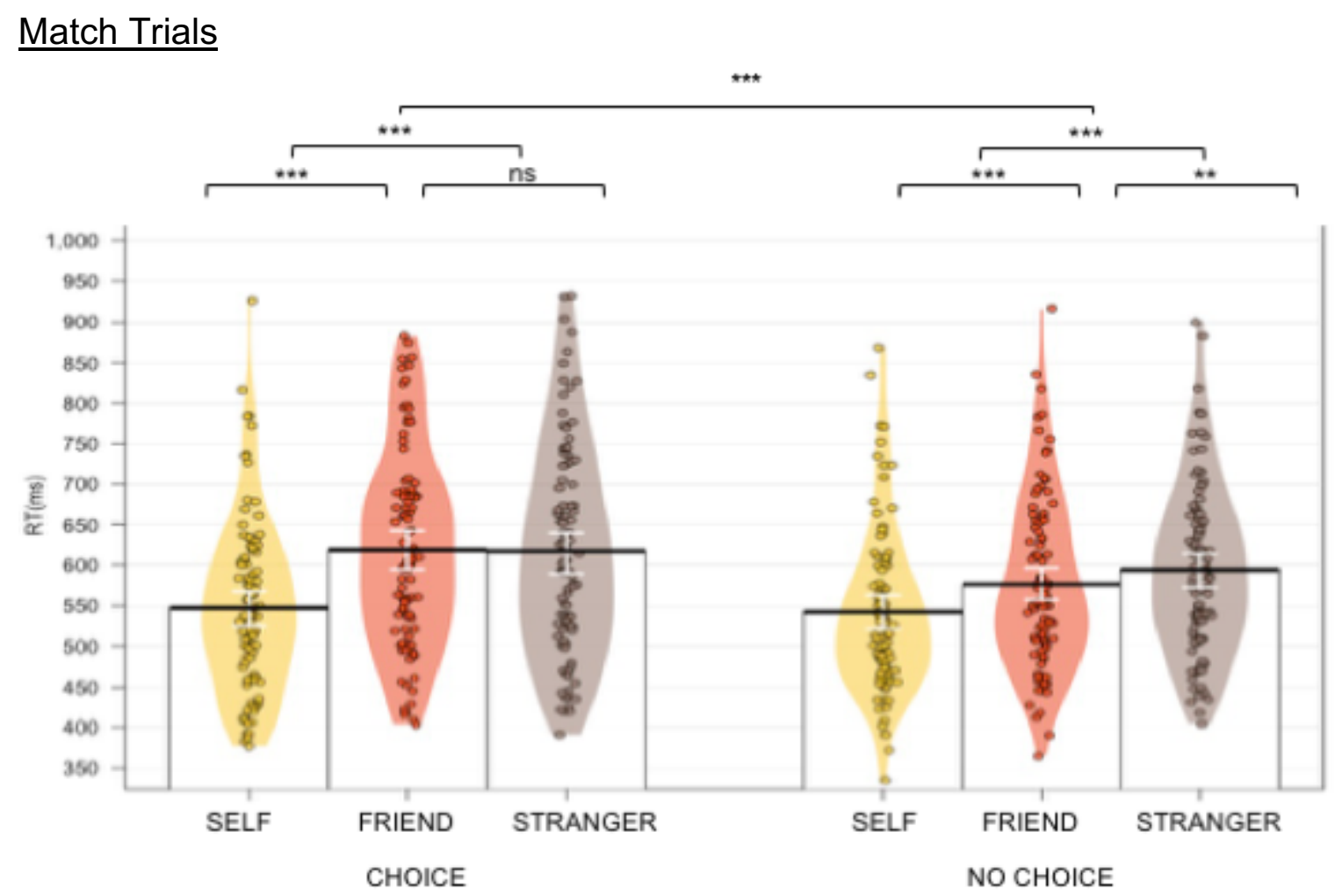

Figure 3. Experiment 3: Mean reaction times (RT) as a function of the voice identity (self, friend, or stranger) and choice (choice vs no choice i.e. whether the self-voice is self-selected or randomly allocated). Graph models match trials only. The error bars indicate the SEs of the means. Coloured segments show smoothed density curves for the full data distribution, while individual dots indicate mean RTs per participant. Horizontal bars show post-hoc comparisons with asterisks denoting significance within RTs as determined via likelihood ratio tests (see Results). ${ }^{* * *} p<.001$.

Identical analyses were run on RTs in mismatch trials. For the post-hoc tests, alpha was set as .008 after Bonferroni correction for six comparisons. These analyses also showed a significant interaction between voice identity and choice $(X 2(2)=10.45$, $p=.005)$. Reaction times were significantly quicker for the self-voice than for either the friend-voice $(p<.001)$ or the stranger-voice $(p=.001)$, but this self-prioritisation effect was only present when the self-voice was chosen. When the self-voice was randomly allocated, performance did not significantly differ for the self, friend and stranger voices. This result supports the notion that choosing the self-voice does increase the prioritisation of it, thus making it more prominent relative to either of the other voices. 


\section{$\underline{\text { Mismatch Trials }}$}

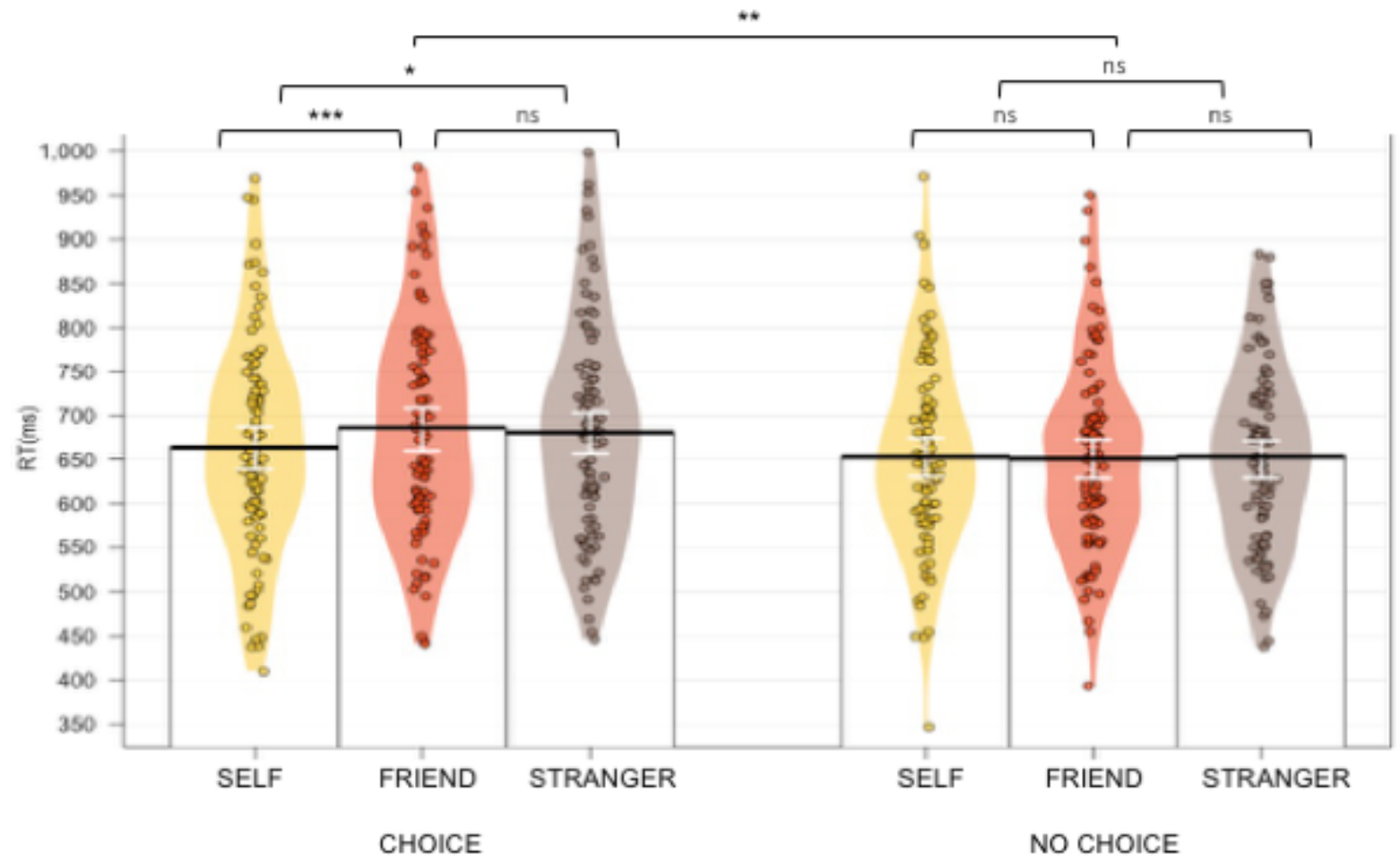

Figure 4. Experiment 3: Mean reaction times (RT) as a function of the voice identity (self, friend, or stranger) and choice (choice vs no choice i.e. whether the self-voice is self-selected or randomly allocated) in mismatch trials only. The error bars indicate the SEs of the means. Coloured segments show smoothed density curves for the full data distribution, while individual dots indicate mean RTs per participant. Horizontal bars show post-hoc comparisons with asterisks denoting significance within RTs as determined via likelihood ratio tests (see Results). ${ }^{* *} p<.001$.

\subsubsection{Sensitivity}

We ran an LMM on d' scores. For the post-hoc tests, alpha was set as .017 after Bonferroni correction for three post-hoc comparisons.

$$
\begin{aligned}
& \text { Imer(sensitivity voice identity * choice } \\
& +(\text { (1|participant), REML = "FALSE") }
\end{aligned}
$$

Table 11. Model output for the analysis of sensitivity in Experiment 3. The reference category for Voice Identity is "Self", the reference category for Choice is "No".

\section{Estimates}


Voice identity (Other)

Main Effect Choice

Choice (Yes)

Interaction Voice Identity * Choice

Voice identity (Friend) * Choice (Yes)

Voice identity (Other) ${ }^{*}$ Choice (Yes)
$-0.44 \quad-0.62--0.26$

$-0.19-0.50-0.12$

$0.24 \quad-0.01-0.49$

$0.11-0.14-0.36$

Full model outputs are reproduced in Table 11. The interaction of voice identity and choice was non-significant $(x 2(2)=3.55, p=.169)$. There was, however, a main effect of voice identity on reaction times $(x 2(2)=34.55, p<.001)$, revealing that $d$-primes for the self-voice were significantly higher than those for the stranger-voice $(p<.001)$ but did not differ significantly from the friend-voice $(p=.020)$. D-prime scores also differed significantly between the friend-voice and other-voice $(p<.001)$. Lastly, we tested for the significance of choice as a fixed effect, however this was non-significant $(X 2(1)=$ $.26, p=.612)$.

\subsubsection{Accuracy}

Finally, we ran GLMMs on trialwise accuracy, first in the match trials. For the post-hoc tests, alpha was set as .008 after Bonferroni correction for six comparisons.

Full model: glmer(accuracy $\sim$ voice identity ${ }^{*}$ choice

$+(1$ participant $)$, family = "binomial")

Table 12. Model output for the analysis of accuracy in Experiment 3.

The reference category for Voice Identity is "Self", the reference category for Choice is "No".

Odd Ratio

18.77

(Intercept)

Main Effect Voice Identity

Voice identity (Friend)

Voice identity (Other)

Main Effect Choice

Choice (Yes)

Interaction Voice Identity * Choice

Voice identity (Friend) * Choice (Yes)

Voice identity (Other) ${ }^{*}$ Choice (Yes)
0.8

0.45

1.13

0.76

1
$\mathrm{Cl}$

$14.76-23.86$

$0.67-0.97$

$0.38-0.53$

$0.81-1.59$

$0.59-0.99$

$0.78-1.28$ 
Full model outputs are reproduced in Table 12. There was an interaction between choice and voice identity $(x 2(2)=6.56, p=.037)$. Pairwise comparisons reveal that when the self-voice was chosen, accuracy for the self-voice becomes significantly greater than accuracy for either the friend-voice $(p<.001)$ or the stranger-voice $(p<.001)$ showing a clear self-prioritisation effect. However, when the self-voice is randomly allocated, accuracy for the self-voice does not differ significantly from the friend-voice $(p=.020)$ though does differ from the stranger-voice $(p<.001)$. Thus, the interaction effect arises from a relative deprioritisation of the friend-voice when the self-voice is chosen compared to when it is not. This pattern of results mirrors the interaction effect found in the RTs: here too, performance for the friend-voice is reduced in the choice condition, leaving the chosen self-voice relatively more prioritised than either of the other two voices. This is not the case when the self-voice is randomly allocated. Lastly, across both groups, accuracy for the friend-voice was significantly higher than accuracy for the stranger-voice (ps<.001).

Identical analyses were also run on accuracy in mismatch trials. For the post-hoc tests, alpha was set as .008 after Bonferroni correction for six comparisons. There was a significant interaction effect here too $(X 2(2)=7.98, p=.018)$. The interaction effect arose from significantly higher accuracy for the self-voice than for the stranger-voice $(p=.005)$ only when the self-voice was chosen. When the self-voice was randomly allocated, performance did not significantly differ for the self, friend and stranger voices. This result supports the notion that choosing the self-voice does increase the prioritisation of it, thus making it more prominent relative to either of the other voices.

\subsection{Exploratory Analyses}

As a means to further explore the implication of choice on self-prioritisation, we indexed the degree of bias for the self-voice (following Sui \& Humphreys, 2017) in both the choice and no choice conditions. A larger score indicates a larger bias, whereas smaller scores indicate a relatively smaller bias. For each participant, a self-bias score can be calculated by taking the difference in RTs for the self-voice versus the friendvoice, dividing it by the sum of RTs across the two conditions, and finally multiplying the result by 100 to achieve a percentage score. A friend-bias score can also be calculated by taking the difference in RT performance for the friend-voice relative to 
the stranger-voice, dividing it by the sum of the RTs across the conditions and multiplying it by 100. Bias scores are plotted in Figure 5.

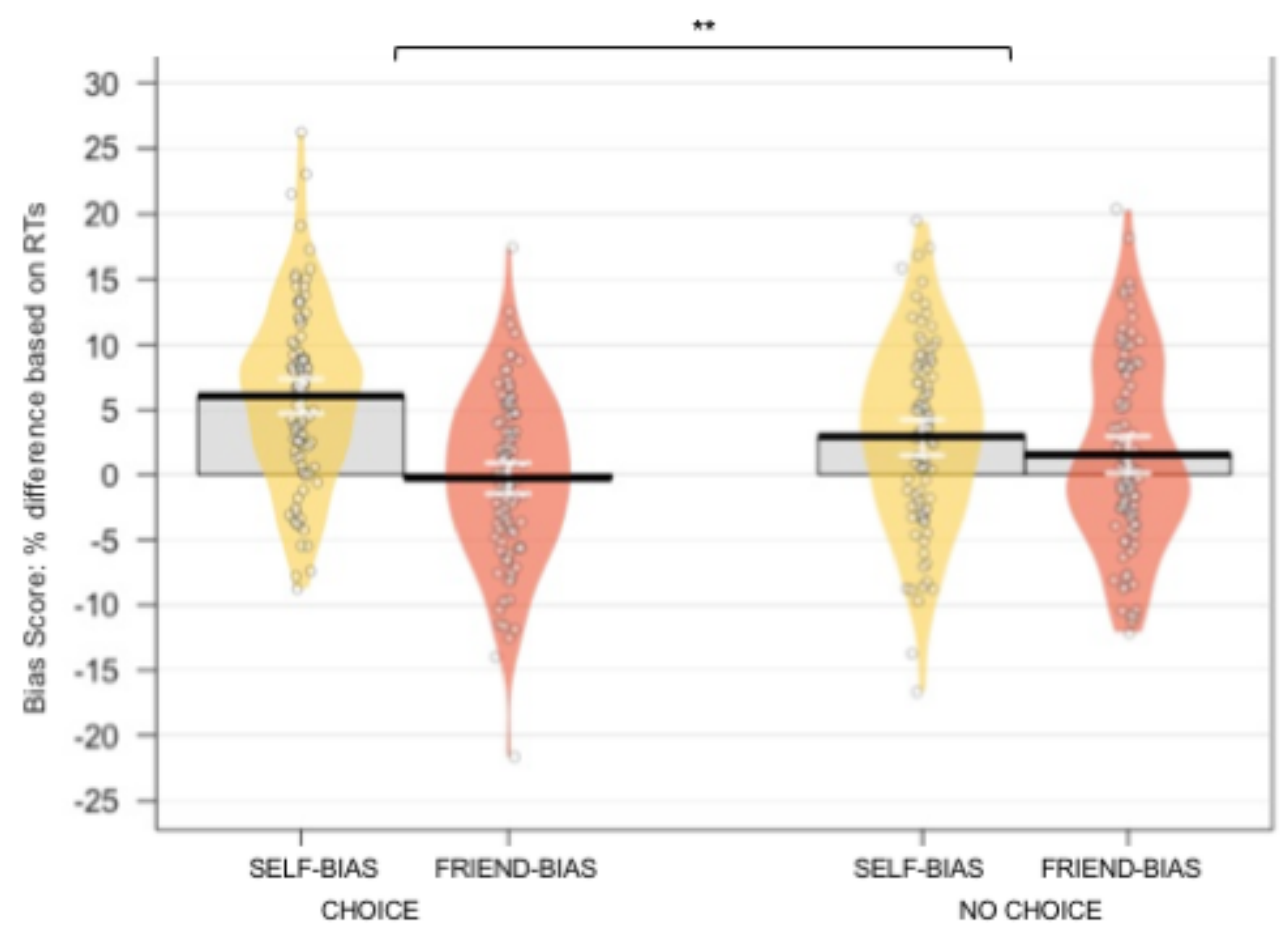

Figure 5. Exploratory analyses, Experiment 3: Measures of bias (self-bias vs. friend-bias) as a function of choice (choice vs. no choice i.e., whether the self-voice is self-selected or randomly allocated). The error bars indicate the SEs of the means. Coloured segments show smoothed density curves for the full data distribution, while individual dots indicate mean scores per participant.

The bias scores show that there is a larger bias - and greater prioritisation - for 'self' relative to 'friend' across both conditions. Importantly though, when the self-voice has been chosen, self-prioritisation increases and the prioritisation of friend decreases relative to when the self-voice has not been chosen. This supports the earlier findings; that there is a greater prominence of self, relative to others, when the stimulus is personally selected. In order to test the significance of this, we calculated the difference between the self-bias and friend-bias score per participant and then ran an independent samples t-test on the resultant scores, comparing the choice vs. no choice groups. This test was significant $(t(190)=-3.05, p=.003)$.

In Experiment 3, we asked whether the self-prioritisation effect could be modulated by choice. Our results show that choice does interact with prioritisation: performance for the friend-voice is significantly reduced as measured by RTs and accuracy - such that it is more distinguished from the self-voice and equivalent to those of the stranger's 
voice - only when the self-voice is chosen. The results suggest that when the selfvoice is chosen, the friend-voice is deemed less socially or personally relevant, and the social distance between self and friend is increased.

\section{General Discussion}

Across 3 experiments, we asked whether an unfamiliar, externally-generated voice could be incorporated into the self and, if so, which factors modulate the resulting processing bias.

In Experiment 1, we showed that an unfamiliar voice could indeed be incorporated into the self, as measured by quicker reaction times, higher accuracy, and increased perceptual sensitivity to the new self-voice relative to a friend or stranger's voice, thus demonstrating a clear self-prioritisation effect. These results are a first demonstration that a voice does not need to be self-generated in order to become integrated into our representation of self and thus be prioritised in perception as a self-relevant stimulus.

This finding is important when considering people who are unable to use their own, self-generated voice to communicate. Specifically, our results support the notion that an alternative vocal signal could quickly become personally relevant as a means of self-expression. Given that reports from AAC users often suggest that the loss of selfvoice can lead to a loss of self-identity (Nathanson, 2017), ways of incorporating a new self-voice into their current representation of self are of direct importance.

In Experiment 2, we began to explore which factors might strengthen this association between the self and a new self-voice, asking whether self-prioritisation would be increased when the self-voice was gender-matched to the identity of the listener.

Contrary to our hypothesis, however, the results suggest that gender-matching the new self-voice to the listener does not significantly moderate prioritisation of that voice: reaction times and perceptual sensitivity did not differ between gender-matched selfvoices and non-gender-matched self-voices. Instead, a significant self-prioritsation was evident in both groups for both RTs and accuracy. 
Given how important voices are to representing our self (McGettigan, 2015), it might be surprising that a more personalised means of vocal expression did not generate a stronger bias, or greater prioritisation. In the memory literature, for instance, Cunningham et al. (2011) suggests that the strength of an item's encompassment within the self should, intuitively, be moderated by how far the item is autobiographically consistent and by how far it matches personal tastes and goals.

Oyserman et al. (2017) too suggest that processing resources are preferentially allocated to identity-consistent stimuli since people prefer to act and make decisions according to what is identity-congruent. Within the current study, however, male and female participants showed a self-prioritisation effect for a self-voice that was not - at least in gender - consistent with their self-representation. It is possible that in this context, the self-voice fulfils the same task demands regardless of gender-matching the perceptual matching task does not require social interaction in which the new selfvoice is used as a primary means of sharing or representing the self-identity. A context demanding the use of the new self-voice as a means to communicate may be required in order for a gender-matched voice to be significantly more relevant to the self than a mismatched voice. More communicative paradigms might shift the bias afforded to the self-voice from that of a merely self-owned stimulus to one of high social salience, due to the critical role it can play in the representation of self and to achieving communicative goals (McGettigan, 2015).

Two key questions remain; first, whether prioritisation can ever be modulated by a stronger association between a stimulus and the self and; second, whether this association can be formed by the way in which the stimulus is encoded to self.

In Experiment 3, we therefore asked whether the strength of the association between the self and a new voice - and so the prioritisation of that voice - would be increased if the participant had made a personal choice to take ownership of it. Specifically, we examined whether listeners showed quicker reaction times and higher accuracy to the self-voice - relative to the friend-voice and stranger-voice - when the self-voice was chosen by the listener as compared to when the self-voice was randomly allocated. 
Our results show that having a choice over the self-voice does interact with perceptual prioritisation. However, the shift in prioritisation did not manifest as quicker reaction times and increased perceptual sensitivity to the chosen self-voice relative to the unchosen self-voice. The interaction arises instead due to reduced performance for the friend-voice when the self-voice was chosen relative to when it was randomly allocated. That is, when the self-voice is chosen, the friend-voice is processed as if it were a stranger's voice, with equivalent RTs for friend and stranger. Conversely, when the self-voice is randomly allocated, there remains a distinction between friend and stranger's voices. Therefore, only when the self-voice is chosen does the distance between the self-voice and the other two voices increase, such that the self-voice becomes more prominent because of reduced prioritisation of the other voices.

The precise manifestation of the effect of choice in Experiment 3 is intriguing. While clearly indicating that choice increased the perceptual separation of the self-voice from the other identities, this seems to have happened via "othering" of the friend voice rather than enhanced prioritisation of the self-voice per se. As there is no prior report of the effect of choice on self-prioritisation within a perceptual matching paradigm, we cannot tell if the effect observed here might be particular to voices or indeed would be found using any category of stimuli (e.g. visual objects) - it will be important to address the generalisability of the finding in future work. It will also be relevant to explore the interplay of mere ownership versus agency (i.e. choosing to take ownership) in this paradigm. Using different tasks, previous studies have shown that while self-bias is most reliably observed when items are both owned and chosen by the participant, even if those items are chosen for another person (Huang et al., 2009). The current paradigm with voice could be extended with a manipulation of which voice is chosen, for example with some participants selecting the voice to represent themselves, and some choosing the friend-voice.

In our study, we have begun to qualify which factors could be harnessed to aid the incorporation of a new, externally-generated voice within the self. We have shown that a new voice can be incorporated into the self after only brief exposure, as evidenced by a self-prioritisation effect in all three experiments. Moreover, personal choice in picking the new self-voice may modulate the distinctiveness of that voice relative to others. Finally, although matching the new self-voice to the gender identity of the 
listener did not seem to modulate prioritisation of it, it is possible that the absence of an effect is due to the lack of a need to use this voice to represent oneself socially within the task. Given that the voice is a highly salient social stimulus, a more immersive, social interaction-based paradigm, in which the new self-voice is actively used to achieve communicated goals, could elicit a stronger bias than we have shown here. In such a context, prioritisation may be modulated by how closely the vocal stimulus can represent the self, including the gender identity of the speaker.

The current results support the case for more personalised voices in which the speaker has agency in choosing their new auditory identity. Future studies should utilise more immersive communicative paradigms to more closely model the adoption of new voices in patients. These studies could better measure the integration of a new voice into the self, the factors that may facilitate integration, and the subsequent bias that is afforded to a new self-voice as a primary means of representing self-identity. These proposed future studies can proceed, importantly, resting on the evidence presented here for the first time; it is possible to incorporate a new voice into our self-identity that would otherwise have been perceived as belonging to an 'other'. We can do this after only brief exposure to it and can enhance our bias towards it through making a personal choice to own it. 


\section{References}

Allan, K., Morson, S., Dixon, S., Martin, D., \& Cunningham, S. (2017). Simulationbased mentalizing generates a "proxy" self-reference effect in memory. Quarterly Journal of Experimental Psychology, 70(6), 1074-1084. doi: 10.1080/17470218.2016.1209532

Anwyl-Irvine, A., Massonnié, J., Flitton, A., Kirkham, N., \& Evershed, J. (2019). Gorilla in our Midst: Gorilla.sc, a new web-based experiment builder. Behavior Research Methods. https://doi.org/10.3758/s13428-01901237-x

Audacity Team. (2019). Audacity(R): Free Audio Editor and Recorder [Computer application]. Version 2.3.2 retrieved March 2019 from https://audacityteam.org/

Bates, D., Maechler, M., Bolker, B., \& Walker, S. (2014). Ime4: Linear mixed-effects models using Eigen and S4. Journal of Statistical Software. 67, 1-23. doi: 10.18637/jss.v067.i01

Benoit, R. G., Gilbert, S. J., Volle, E., \& Burgess, P. W. (2010). When I think about me and simulate you: medial rostral prefrontal cortex and self-referential processes. Neuroimage $\quad$ 50, 1340-1349. doi:10.1016/j.neuroimage.2009.12.091

Boersma, P., \& Weenink, D. (2018). Praat: doing phonetics by computer [Computer program].

Cloutier, J., \& Macrae, C. (2008). The feeling of choosing: Self-involvement and the cognitive status of things past. Consciousness and Cognition, 17(1), 125-135. doi: 10.1016/j.concog.2007.05.010

Cunningham, S. J., Turk, D. J., Macdonald, L. M., \& Macrae, C. N. (2008). Yours or mine? Ownership and memory. Consciousness and Cognition, 17, 312-318. doi:10.1016/j.concog.2007.04.003

Cunningham, S., Brady-Van den Bos, M., \& Turk, D. (2011). Exploring the effects of ownership and choice on self-memory biases. Memory, 19(5), 449-461. doi: 10.1080/09658211.2011.584388 
Gregg, A., Mahadevan, N., \& Sedikides, C. (2017). The SPOT effect: people spontaneously prefer their own theories. Quarterly Journal of Experimental Psychology, 70(6), 996-1010. doi: 10.1080/17470218.2015.1099162

Holzman, P. S., \& Rousey, C. (1966). The voice as a percept. Journal of Personality and Social Psychology, 4(1), 79-86. doi: 10.1037/h0023518

Huang, Y., Wang, L., \& Shi, J. (2009). When do objects become more attractive? The individual and interactive effects of choice and ownership on object evaluation. Personality and Social Psychology Bulletin, 35(6), 713-722. doi: 10.1177/0146167209333046

Hughes, S., \& Harrison, M. (2013). I like my voice better: self-enhancement bias in perceptions of voice attractiveness. Perception, 42(9), 941-949. doi: $10.1068 / p 7526$

Hughes, S. M., Mogilski, J. K., \& Harrison, M. A. (2014). The perception and parameters of intentional voice manipulation. Journal of Nonverbal Behavior, 38(1), 107-127. doi: 10.1007/s10919-013-0163-z

Jones, J., Pelham, B., Carvallo, M., \& Mirenberg, M. (2004). How do I love thee? Let me count the Js: Implicit egotism and interpersonal attraction. Journal of Personality and Social Psychology, 87(5), 665-683. doi: 10.1037/00223514.87.5.665

Kreiman, J., \& Sidtis, D. (2011). Foundations of Voice Studies: An Interdisciplinary Approach to Voice Production and Perception. WileyBlackwell. https://doi.org/10.1002/9781444395068

Lass, N. J., Hughes, K. R., Bowyer, M. D., Waters, L. T., \& Bourne, V. T. (1976). Speaker sex identification from voiced, whispered, and filtered isolated vowels. The Journal of the Acoustical Society of America, 59(3), 675-678.

Lenth, R. (2016) Least-Squares Means: The R Package Ismeans. Journal of Statistical Software, 69(1), 1-33. doi:10.18637/jss.v069.i01 
McGettigan, C., Eisner, F., Agnew, Z. K., Manly, T., Wisbey, D., \& Scott, S. K. (2013). T'ain't what you say, it's the way that you say it-left insula and inferior frontal cortex work in interaction with superior temporal regions to control the performance of vocal impersonations. Journal of Cognitive Neuroscience. 25, 1875-1886. doi: 10.1162/jocn_a_00427

McGettigan, C., \& Scott, S. K. (2014). Voluntary and involuntary processes affect the production of verbal and non-verbal signals by the human voice. Behavioral and Brain Sciences. 37, 564-565. doi: 10.1017/S0140525X13004123

McGettigan, C. (2015). The social life of voices: studying the neural bases for the expression and perception of the self and others during spoken communication. Frontiers In Human Neuroscience, 9. doi: 10.3389/fnhum.2015.00129

Nathanson, E. (2017). Native voice, self-concept and the moral case for personalized voice technology. Disability and Rehabilitation, 39(1), 73-81. doi: 10.3109/09638288.2016.1139193

Owren, M., Berkowitz, M., \& Bachorowski, J. (2007). Listeners judge talker sex more efficiently from male than from female vowels. Perception \& Psychophysics, 69(6), 930-941. doi: 10.3758/bf03193930

Oyserman, D., Lewis, N., Yan, V., Fisher, O., O'Donnell, S., \& Horowitz, E. (2017). An Identity-Based Motivation Framework for Self-Regulation. Psychological Inquiry, 28(2-3), 139-147. doi: 10.1080/1047840x.2017.1337406

Payne, S., Tsakiris, M., \& Maister, L. (2017). Can the self become another? Investigating the effects of self-association with a new facial identity. Quarterly Journal of Experimental Psychology, 70(6), 1085-1097. doi: $10.1080 / 17470218.2015 .1137329$

Pohlert, T. (2014). The pairwise comparison of mean ranks package (PMCMR). R Package, https://CRAN.R-project.org/package=PMCMR

Puts, D., Hill, A., Bailey, D., Walker, R., Rendall, D., \& Wheatley, J. et al. (2016). Sexual selection on male vocal fundamental frequency in humans and other 
anthropoids. Proceedings Of The Royal Society B: Biological Sciences, 283(1829), 20152830. doi: 10.1098/rspb.2015.2830

$\mathrm{R}$ Core Team. R: A language and environment for statistical computing ( $R$ Foundation for Statistical Computing, Vienna, Austria, 2013). http://www.Rproject.org/.

Schäfer, S., Wesslein, A., Spence, C., Wentura, D., \& Frings, C. (2016). Selfprioritization in vision, audition, and touch. Experimental Brain Research, 234(8), 2141-2150. doi: 10.1007/s00221-016-4616-6

Stanislaw, H., \& Todorov, N. (1999) Calculation of signal detection theory measures. Behavior Research Methods, Instruments, and Computers, 31:137-149. doi:10.3758/BF03207704

Sui, J., \& Humphreys, G. (2017). Aging enhances cognitive biases to friends but not the self. Psychonomic Bulletin \& Review, 24(6), 2021-2030. doi: $10.3758 / \mathrm{s} 13423-017-1264-1$

Sui, J., Rotshtein, P., \& Humphreys, G. W. (2013). Coupling social attention to the self forms a network for personal significance. Proceedings of the National Academy of Sciences, 110, 7607-7612. doi: 10.1073/pnas.1221862110

Sui, J., He, X., \& Humphreys, G. W. (2012). Perceptual effects of social salience: Evidence from self-prioritization effects on perceptual matching. Journal of Experimental Psychology: Human Perception and Performance, 38, 11051117. doi:10.1037/ a0029792

Symons, C. S., \& Johnson, B. T. (1997). The self-reference effect in memory: A metaanalysis. Psychological Bulletin, 121, 371-394. doi:10.1037/00332909.121.3.371

Woods, K. J., Siegel, M. H., Traer, J., \& McDermott, J. H. (2017). Headphone screening to facilitate web-based auditory experiments. Attention, Perception, and Psychophysics. 79, 2064-2072. 10.3758/s13414-017-1361- 


\section{SUPPLEMENTAL MATERIAL}

1. Analyses of Variance for Experiments 1-3 RTs

\section{Experiment 1:}

(i) RTs

The RT data were submitted to a 3 ('voice identity': self vs. friend vs. stranger) $\times 2$ ('trial type': match vs. mismatch) within-subject ANOVA to determine whether there were statistically significant differences in reaction times for the self-voice, friend-voice and stranger-voice. There were no outliers, as assessed by examination of studentised residuals for values greater than \pm 3 , and reaction time data was normally distributed, as assessed by Shapiro-Wilk's test of normality on the studentised residuals $(p>.05)$.

The main effect of trial type was significant, $F(1,30)=172.59, p<.001, \eta^{2} p=.85$ and there was a significant voice identity $x$ trial type interaction, $F(2,60)=6.878, p=.002$, $\eta^{2} p=.18$. As this suggests that the RT differences for the condition differed for match and mismatch trials, we analysed match and mismatch trials separately following Sui and colleagues' logic (2012). Specifically, in a one-way ANOVA with match trials, the data showed a significant effect of the voice identity condition, $F(1,30)=81.174, p=$ $<.001, \eta^{2}=.730$, with post hoc comparisons showing that average RTs for the selfvoice were significantly quicker than RTs for both the friend-voice $(p=.02)$ and stranger-voice $(p=.009)$, with Bonferroni correction for multiple comparisons. These results show a significant self-prioritisation effect in the RTs for the self-voice. In contrast, there was no significant effect of condition for the mismatch pairs $(p=0.86)$.

\section{(ii) Sensitivity}

The indices of d' were analysed using a one-way repeated measures ANOVA to determine whether there were statistically significant differences in perceptual sensitivity according to the association (either self, friend, or stranger). There were no outliers, the data was normally distributed as assessed by a boxplot examination and Shapiro-Wilk test $(p>.05)$, and the assumption of sphericity was met. 
The resulting $d^{\prime}$ indices were submitted to a one-way ANOVA with three levels (voice identity: self vs. friend vs. stranger). The relevant Helmert contrast (Schäfer et al., 2016), comparing d' for the self-voice to d' for both the friend-voice and stranger-voice was significant, $F(1,30)=5.943, p=.021, \eta 2 p=.16$, indicating a significant selfprioritisation effect in sensitivity measures.

\section{Experiment 2:}

(i) $\mathrm{RTs}$

The RT data from matched trials were submitted to a 3 ('voice identity': self vs. friend vs. stranger) $\times 2$ ('gender-matching': gender-matched vs. non-gender-matched) mixed ANOVA to determine whether there were statistically significant differences in reaction times for the self-voice, friend-voice and stranger-voice according to gender-matching.

The interaction between voice identity and gender-matching was non-significant, however, the main effect of voice identity was significant, $F(2,188)=16.60, p<.001$. Planned contrasts reveal that there was a significant Helmert contrast for the selfvoice, $F(1,194)=27.16, p<.001$, such that reaction times to the self-voice were significantly quicker than for either the friend or stranger voice.

Results from identical analyses of RT data within the mismatch trials were all nonsignificant.

\section{(ii) Sensitivity}

The $d$ ' indices were submitted to a 3 ('voice identity': self vs. friend vs. stranger) $\times 2$ ('gender-matching': gender-matched vs. non-gender-matched) mixed ANOVA. The results showed that the interaction between gender-matching and voice identity was non-significant, thereby mirroring the RT results. However, the main effect of voice identity was significant $F(2,188)=8.57, p=<.001$. Planned contrasts revealed a significant Helmert contrast for the self-voice $F(1,94)=6.17, p=.015$, showing that accuracy for the self-voice was significantly greater than accuracy for friend-voice and stranger-voice. 


\section{Experiment 3:}

(i) RTs

The RT data from matched trials were submitted to a 3 ('voice identity': self vs. friend vs. stranger) $\times 2$ ('choice': chosen vs. not chosen) mixed ANOVA to determine whether there were statistically significant differences in reaction times for the self-voice, friendvoice and stranger-voice according to choice.

The interaction between voice identity and choice was significant $F(2,380)=4.48$, $p=.012$, with planned contrasts showing there is a significant Helmert contrast for the self-voice, $F(2,190)=6.19, p=.014)$, such that reaction times to the self-voice were significantly quicker than those for either the friend-voice or stranger voice in the choice group, but not in the no choice group. This indicates a significant selfprioritisation effect for the self-voice only in the choice group.

The RT data from mismatch trials were then submitted to a 3 ('voice identity': self vs. friend vs. stranger) $\times 2$ ('choice': chosen vs. not chosen) mixed ANOVA. The interaction between voice identity and choice was significant, $F(2,380)=3.03, p=.05$, with planned contrasts showing there was a significant Helmert contrast for the selfvoice, $F(1,190)=4.70, p=.031)$, such that reaction times to the self-voice were significantly quicker than those for either the friend-voice or stranger voice in the choice group, but not in the no choice group. This indicates a significant selfprioritisation effect for the self-voice only in the choice group.

\section{(ii) Sensitivity}

The resulting $d^{\prime}$ ' indices were submitted to a 3 ('voice identity': self vs. friend vs. stranger) $\times 2$ ('choice': chosen vs. not chosen) mixed ANOVA.

The results showed that the interaction between voice identity and choice was nonsignificant but there was a significant main effect of voice identity, $F(2,380)=18.05$, $p<.001$. Planned contrasts reveal that there was a significant Helmert contrast for the self-voice, $F(1,190)=24.47, p<.001$, such that perceptual sensitivity to the self-voice was significantly greater than for either the friend or stranger voice. 


\section{Pilot: Voice Ratings}

The figures below show the plotted pilot data for ratings of attractiveness and trustworthiness across 12 different voices $(6 \mathrm{M}, 6 \mathrm{~F})$.

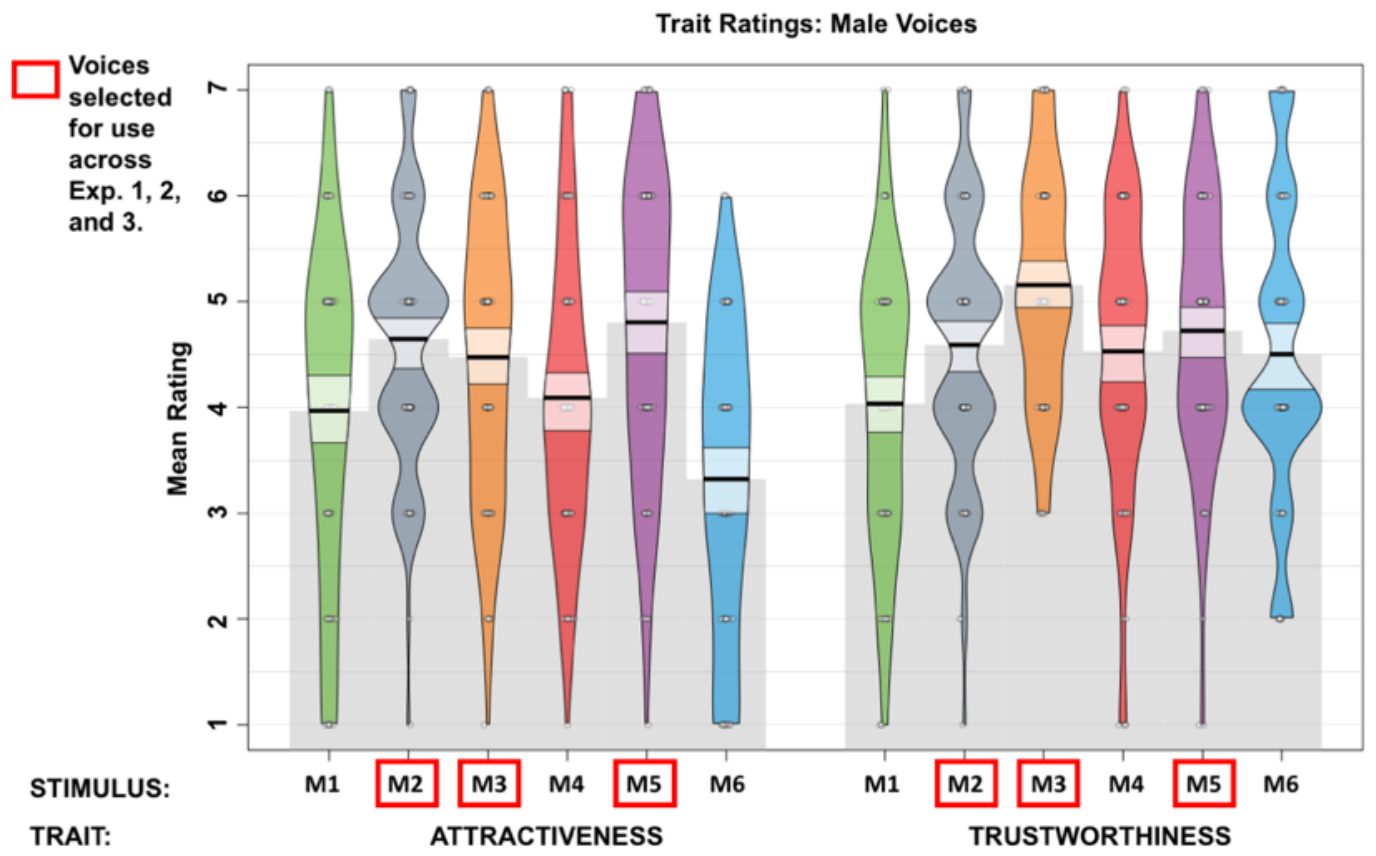

Trait Ratings: Female Voices

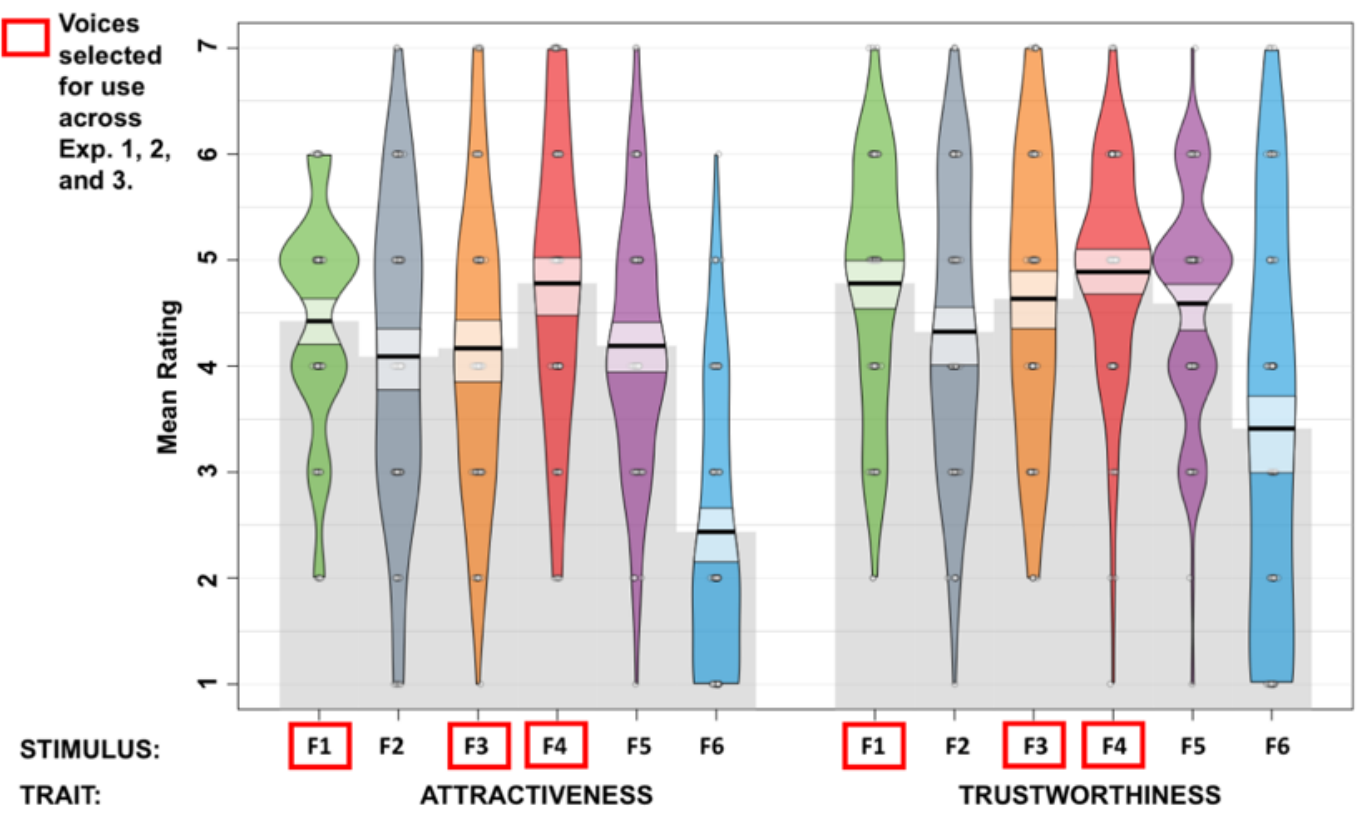


3. Acoustic analysis of voice stimuli used across experiments 1-3.

\begin{tabular}{|c|c|c|c|c|c|c|c|c|c|c|c|}
\hline Stimulus* & $\begin{array}{c}\text { Duratio } \\
n\end{array}$ & $\begin{array}{c}\text { F0 } \\
\text { (mean) }\end{array}$ & $\begin{array}{l}\text { Fo } \\
\text { (sd) }\end{array}$ & $\begin{array}{c}\text { Fo } \\
\text { (min) }\end{array}$ & $\begin{array}{c}\text { F0 } \\
(\max )\end{array}$ & F1 & F2 & F3 & F4 & F5 & $\begin{array}{c}\text { HNR } \\
\text { (mean) }\end{array}$ \\
\hline F1_t1 & 0.31 & 187.89 & 14.29 & 175.89 & 220.45 & 639.53 & 1617.03 & 2714.92 & 3702.50 & 4128.69 & 12.22 \\
\hline F1_t2 & 0.31 & 178.58 & 9.38 & 157.36 & 196.32 & 642.28 & 1636.51 & 2652.49 & 3495.60 & 4263.88 & 11.01 \\
\hline $\mathrm{F} 2 \_\mathrm{t} 1$ & 0.51 & 171.66 & 14.94 & 150.20 & 207.37 & 646.41 & 1652.70 & 2809.46 & 3696.54 & 4557.76 & 15.57 \\
\hline F2_t2 & 0.61 & 164.58 & 11.03 & 147.55 & 194.57 & 701.31 & 1795.87 & 2828.55 & 3806.78 & 4556.72 & 17.41 \\
\hline F3_t1 & 0.43 & 196.49 & 14.34 & 143.81 & 225.23 & 507.47 & 1622.05 & 2648.29 & 3855.38 & 4777.21 & 17.39 \\
\hline F3_t2 & 0.41 & 196.26 & 19.86 & 134.31 & 224.84 & 473.30 & 1631.49 & 2589.09 & 3778.98 & 4860.65 & 18.20 \\
\hline M1_t1 & 0.57 & 116.09 & 31.06 & 92.72 & 198.63 & 891.34 & 1855.35 & 2934.17 & 4064.00 & 5010.89 & 8.66 \\
\hline M1_t2 & 0.68 & 102.67 & 6.47 & 94.47 & 114.56 & 1082.90 & 2056.04 & 3107.44 & 4147.03 & 4872.03 & 9.31 \\
\hline M2_t1 & 0.43 & 123.96 & 42.78 & 72.64 & 195.41 & 666.08 & 1650.48 & 2850.66 & 3821.53 & 4276.41 & 8.35 \\
\hline M2_t2 & 0.33 & 119.73 & 26.42 & 74.49 & 147.51 & 507.46 & 1457.87 & 2685.39 & 3637.41 & 4129.73 & 8.79 \\
\hline M3_t1 & 0.30 & 116.34 & 16.74 & 99.06 & 154.79 & 553.75 & 1595.84 & 2630.81 & 3545.99 & 4536.50 & 9.23 \\
\hline M3_t2 & 0.26 & 120.36 & 16.78 & 96.54 & 152.42 & 511.46 & 1571.77 & 2638.38 & 3522.79 & 4471.77 & 7.09 \\
\hline
\end{tabular}

